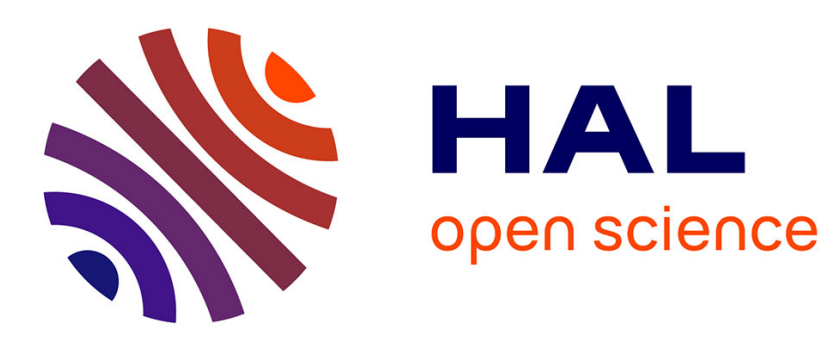

\title{
Bloch's conjecture for certain hyperkähler fourfolds
}

Robert Laterveer

\section{To cite this version:}

Robert Laterveer. Bloch's conjecture for certain hyperkähler fourfolds. Pure and Applied Mathematics Quarterly, 2017, 13 (4), pp.639-692. 10.4310/PAMQ.2017.v13.n4.a3 . hal-02310261

\section{HAL Id: hal-02310261 https://hal.science/hal-02310261}

Submitted on 10 Oct 2019

HAL is a multi-disciplinary open access archive for the deposit and dissemination of scientific research documents, whether they are published or not. The documents may come from teaching and research institutions in France or abroad, or from public or private research centers.
L'archive ouverte pluridisciplinaire HAL, est destinée au dépôt et à la diffusion de documents scientifiques de niveau recherche, publiés ou non, émanant des établissements d'enseignement et de recherche français ou étrangers, des laboratoires publics ou privés. 


\title{
BLOCH'S CONJECTURE FOR CERTAIN HYPERKÄHLER FOURFOLDS, AND EPW SEXTICS
}

\author{
ROBERT LATERVEER
}

\begin{abstract}
On a hyperkähler fourfold $X$, Bloch's conjecture predicts that any involution acts trivially on the deepest level of the Bloch-Beilinson filtration on the Chow group of 0-cycles. We prove a version of Bloch's conjecture when $X$ is the Hilbert scheme of 2 points on a generic quartic in $\mathbb{P}^{3}$, and the involution is the non-natural, non-symplectic involution on $X$ constructed by Beauville. This has consequences for the Chow groups of certain EPW sextics.
\end{abstract}

\section{INTRODUCTION}

For a smooth projective variety $X$ over $\mathbb{C}$, let $A^{i}(X):=C H^{i}(X)_{\mathbb{Q}}$ denote the Chow group of codimension $i$ algebraic cycles modulo rational equivalence with $\mathbb{Q}$-coefficients. Let $A_{\text {hom }}^{i}(X)$ and $A_{A J}^{i}(X) \subset A^{i}(X)$ denote the subgroups of homologically trivial (resp. Abel-Jacobi trivial) cycles. Notoriously, Chow groups of codimension $i>1$ cycles are still but poorly understood. To cite one prominent example, there is Bloch's conjecture (which even for surfaces of geometric genus 0 remains stubbornly conjectural, reminiscent of a castle lying under siege for many years but showing no intention of being ready to hoist the white flag of complete surrender):

Conjecture 1.1 (Bloch [5]). Let $X$ be a smooth projective variety of dimension $n$. Let $\Gamma \in$ $A^{n}(X \times X)$ be a correspondence such that

$$
\Gamma^{*}=0: \quad H^{p}\left(X, \mathcal{O}_{X}\right) \rightarrow H^{p}\left(X, \mathcal{O}_{X}\right) \text { for all } p>0 .
$$

Then

$$
\Gamma^{*}=0: \quad A_{\text {hom }}^{n}(X) \rightarrow A_{\text {hom }}^{n}(X) .
$$

One could also state a variant of Bloch's conjecture for codimension 2 cycles:

Conjecture 1.2. Let $X$ be a smooth projective variety of dimension $n$. Let $\Gamma \in A^{n}(X \times X)$ be a correspondence such that

$$
\Gamma^{*}=0: \quad H^{2}\left(X, \mathcal{O}_{X}\right) \rightarrow H^{2}\left(X, \mathcal{O}_{X}\right) .
$$

Then

$$
\Gamma^{*}=0: \quad A_{A J}^{2}(X) \rightarrow A_{A J}^{2}(X) .
$$

2010 Mathematics Subject Classification. Primary 14C15, 14C25, 14C30.

Key words and phrases. Algebraic cycles, Chow groups, motives, Bloch's conjecture, Bloch-Beilinson filtration, hyperkähler varieties, K3 surfaces, Hilbert schemes, EPW sextics, double EPW sextics, non-symplectic involution, multiplicative Chow-Künneth decomposition, "spread" of algebraic cycles in a family. 
Now, let us restrict our focus to the realm of hyperkähler varieties (by which we mean: projective irreducible holomorphic symplectic manifolds [2]). In this case, $H^{*}\left(X, \mathcal{O}_{X}\right)$ is generated by $H^{2}\left(X, \mathcal{O}_{X}\right)$ which is of dimension 1 , and so conjecture 1.1 takes on a particularly appealing form:

Conjecture 1.3. Let $X$ be a hyperkähler variety of dimension $n$. Let $\omega \in H^{2,0}(X)$ be a holomorphic 2-form. Let $\Gamma \in A^{n}(X \times X)$ be a correspondence such that

$$
\Gamma^{*}\left(\omega^{r}\right)=0 \text { for all } r>0 \text {. }
$$

Then

$$
\Gamma^{*}=0: \quad A_{\text {hom }}^{n}(X) \rightarrow A_{\text {hom }}^{n}(X) .
$$

We also get the following particular case:

Conjecture 1.4. Let $X$ be a hyperkähler variety of dimension $n=4 m$ (where $m \in \mathbb{N}$ ). Let $\iota \in \operatorname{Aut}(X)$ be an involution. Then

$$
\iota^{*}=\mathrm{id}: \quad F^{n} A^{n}(X) \rightarrow F^{n} A^{n}(X) .
$$

Here $F^{n} A^{n}(X)$ denotes the "deepest level" of the Bloch-Beilinson filtration, which conjecturally exists for all smooth projective varieties [18], [19], [20], [25], [26], and for which good candidates are known to exist unconditionally for certain hyperkähler varieties [35], [46]. (The point of conjecture 1.4 is that the action of $\iota$ on $F^{n} A^{n}(X)$ is conjecturally determined by the action of $\iota$ on $H^{n, 0}(X)=H^{4 m, 0}(X)$, which is the identity.)

In dimension $n=2$, certain cases of conjecture 1.3 have been proven:

Theorem 1.5 (Huybrechts [17], Voisin [41]). Let $X$ be a K3 surface. Let $f \in \operatorname{Aut}(X)$ be a finite order automorphism that is symplectic. Then

$$
f^{*}=\mathrm{id}: \quad A^{2}(X) \rightarrow A^{2}(X) .
$$

That is, conjecture 1.3 is true when $X$ is a $K 3$ surface and $\Gamma=\Gamma_{f}-\Delta_{X}$ (where $\Gamma_{f}$ denotes the graph of $f$, and $f$ is as in theorem 1.5).

In dimension $n>2$, certain cases of conjecture 1.3 have been proven for the Fano variety of lines on a cubic fourfold [15]. There is also a result for what is perhaps the prime series of examples of hyperkähler fourfolds: the Hilbert scheme $S^{[2]}$ of 2 points on a $K 3$ surface $S$ [35, Proposition 5.2]:

Theorem 1.6 (Shen-Vial [35]). Let $S$ be a K3 surface, and let $X=S^{[2]}$. Let $f \in \operatorname{Aut}(X)$ be a natural automorphism of finite order that is symplectic. Then

$$
\begin{array}{ll}
f^{*}=\mathrm{id}: & A^{4}(X) \rightarrow A^{4}(X), \\
f^{*}=\mathrm{id}: & A_{\text {hom }}^{2}(X) \rightarrow A_{\text {hom }}^{2}(X) .
\end{array}
$$

That is, conjecture 1.3 is true for $X=S^{[2]}$ and $\Gamma=\Gamma_{f}-\Delta_{X}$.

Here, a natural automorphism is by definition an automorphism of $X$ that is induced by an automorphism of $S$. Theorem 1.6 is proven by reducing to theorem 1.5. The goal of this article is 
to go beyond theorem 1.6, by also considering non-natural and non-symplectic automorphisms of $S^{[2]}$.

Let $X=S^{[2]}$ be a Hilbert scheme with $S$ a $K 3$ surface, and assume that $X$ has an antisymplectic involution $\iota$. The involution $\iota$, being anti-symplectic, has the property that

$$
\iota^{*}=-\mathrm{id}: \quad H^{2}\left(X, \mathcal{O}_{X}\right) \rightarrow H^{2}\left(X, \mathcal{O}_{X}\right) .
$$

Conjecture 1.2 thus predicts that

$$
\iota \stackrel{? ?}{=}-\mathrm{id}: \quad A_{\text {hom }}^{2}(X) \rightarrow A_{\text {hom }}^{2}(X) .
$$

Can one prove this conjectural equality?

One classical case where an anti-symplectic involution exists is that of the Hilbert scheme $X=S^{[2]}$, where $S \subset \mathbb{P}^{3}$ is a smooth quartic with Picard number $\rho(S)=1$. In this case, it is known ([8], cf. theorem 2.20 below) that the only non-trivial automorphism of $X$ is the non-symplectic, non-natural involution

$$
\iota: X \rightarrow X
$$

which was first studied by Beauville [1]. Our main result implies that in this case, conjecture 1.4 and a weak version of the conjectural equality (1) are true:

Theorem (=theorem 4.1). Let $S \subset \mathbb{P}^{3}$ be a smooth quartic with Picard number $\rho(S)=1$, and let $X=S^{[2]}$. Let $\iota \in \operatorname{Aut}(X)$ be the non-symplectic involution of [1]. Then

$$
\begin{aligned}
\iota^{*}=-\mathrm{id}: & A_{(2)}^{i}(X) \rightarrow A_{(2)}^{i}(X) \text { for } i=2,4 ; \\
\iota^{*}=\mathrm{id}: & A_{(4)}^{4}(X) \rightarrow A_{(4)}^{4}(X) .
\end{aligned}
$$

Here, $A_{(*)}^{*}(X)$ denotes the bigraded ring structure constructed by Shen-Vial [35] using (their version of) the Fourier transform. To establish equality (1) for $X$ as in theorem 4.1, it remains to prove the conjectural equality

$$
A_{(2)}^{2}(X) \stackrel{? ?}{=} A_{h o m}^{2}(X) .
$$

Unfortunately, equality (2) does not seem to be known for any $K 3^{[2]}$. Some evidence for equality (2) is that it is true if there exists a Bloch-Beilinson filtration on $A^{*}(X)$ of which the Fourier decomposition $A_{(*)}^{*}(X)$ is a splitting; more concretely, equality (2) is equivalent to Murre's conjecture $D$ for $X$ [35, Theorem 3.3].

Theorem 4.1 has consequences for certain EPW sextics:

Corollary (=corollary 5.4). Let $X$ and $\iota$ be as in theorem 4.1, and let $Y:=X / \iota$ be the associated EPW sextic. For any $r \in \mathbb{N}$, let

$$
E^{*}\left(Y^{r}\right) \subset A^{*}\left(Y^{r}\right)
$$

be the subring generated by (pullbacks of) $A^{1}(Y)$ and $A^{2}(Y)$. The cycle class map

$$
E^{k}\left(Y^{r}\right) \rightarrow H^{2 k}\left(Y^{r}\right)
$$

is injective for $k \geq 4 r-1$. 
In particular, taking $r=1$, we find that the subspaces

$$
\begin{aligned}
& \operatorname{Im}\left(A^{2}(Y) \otimes A^{1}(Y) \rightarrow A^{3}(Y)\right), \\
& \operatorname{Im}\left(A^{2}(Y) \otimes A^{2}(Y) \rightarrow A^{4}(Y)\right)
\end{aligned}
$$

are of dimension 1 (corollary 5.6). This is analogous to known results for 0 -cycles on $K 3$ surfaces [4] and on certain Calabi-Yau varieties [40], [14] (cf. remark 5.8 below).

To prove theorem 4.1, we employ the technique of "spread" of algebraic cycles in a family, as developed by Voisin in her work on the Bloch/Hodge equivalence for complete intersections [42], [43], [44], [45]. At the heart of our proof is a result of Voisin about the triviality of certain Chow groups of the relative fourfold fibre product of the family of all smooth quartics, provided the (Lefschetz or Voisin) standard conjecture is true ([42, Proposition 4.11], cf. also theorem 4.5 below). The most delicate part of the proof is to circumvent recourse to the standard conjectures in Voisin's result; in this case, this works because we can reduce the problem to a certain relative correspondence of codimension 2 (rather than 4 ).

Another case where an anti-symplectic involution exists on $X=S^{[2]}$ is when $S$ is a degree $2 K 3$ surface (i.e., a double cover of the plane ramified along a smooth sextic). In this case, the anti-symplectic involution is natural (induced by the covering involution of $S$ ), and the statement of theorem 4.1 can be easily proven for this case (cf. proposition 3.1). Other cases where an anti-symplectic involution exists on $X=S^{[2]}$ are when $S$ is a generic $K 3$ of degree 20,26 or 34 (theorem 2.20). Proving the statement of theorem 4.1 for these cases would be interesting, but appears to be difficult (cf. question 6.3).

Conventions. In this article, the word variety will refer to a reduced irreducible scheme of finite type over $\mathbb{C}$. A subvariety is a (possibly reducible) reduced subscheme which is equidimensional.

All Chow groups will be with rational coefficients: we denote by $A_{j}(X)$ the Chow group of $j$-dimensional cycles on $X$ with $\mathbb{Q}$-coefficients; for $X$ smooth of dimension $n$ the notations $A_{j}(X)$ and $A^{n-j}(X)$ will be used interchangeably.

The notations $A_{\text {hom }}^{j}(X), A_{A J}^{j}(X)$ will be used to indicate the subgroups of homologically trivial, resp. Abel-Jacobi trivial cycles. For a morphism $f: X \rightarrow Y$, we will write $\Gamma_{f} \in$ $A_{*}(X \times Y)$ for the graph of $f$. The contravariant category of Chow motives (i.e., pure motives with respect to rational equivalence as in [34], [26]) will be denoted $\mathcal{M}_{\text {rat }}$.

We will write $H^{j}(X)$ to indicate singular cohomology $H^{j}(X, \mathbb{Q})$.

Given an involution $\iota$ on $X$, we will write $A^{j}(X)^{\iota}\left(\right.$ and $\left.H^{j}(X)^{\iota}\right)$ for the subgroup invariant under $\iota$.

\section{PRELIMINARY}

\subsection{Quotient varieties.}

Definition 2.1. A projective quotient variety is a variety

$$
X=Y / G \text {, }
$$

where $Y$ is a smooth projective variety and $G \subset A u t(Y)$ is a finite group. 
Proposition 2.2 (Fulton [16]). Let $X$ be a projective quotient variety of dimension $n$. Let $A^{*}(X)$ denote the operational Chow cohomology ring. The natural map

$$
A^{i}(X) \rightarrow A_{n-i}(X)
$$

is an isomorphism for all $i$.

Proof. This is [16, Example 17.4.10].

Remark 2.3. It follows from proposition 2.2 that the formalism of correspondences goes through unchanged for projective quotient varieties (this is also noted in [16, Example 16.1.13]). We can thus consider motives $(X, p, 0) \in \mathcal{M}_{\text {rat }}$, where $X$ is a projective quotient variety and $p \in$ $A^{n}(X \times X)$ is a projector. For a projective quotient variety $X=Y / G$, one readily proves (using Manin's identity principle) that there is an isomorphism

$$
h(X) \cong h(Y)^{G}:=\left(Y, \Delta_{Y}^{G}, 0\right) \text { in } \mathcal{M}_{\text {rat }},
$$

where $\Delta_{Y}^{G}$ denotes the idempotent $\frac{1}{|G|} \sum_{g \in G} \Gamma_{g}$.

\subsection{MCK decomposition.}

Definition 2.4 (Murre [25]). Let $X$ be a smooth projective variety of dimension $n$. We say that $X$ has a $\mathrm{CK}$ decomposition if there exists a decomposition of the diagonal

$$
\Delta_{X}=\Pi_{0}+\Pi_{1}+\cdots+\Pi_{2 n} \text { in } A^{n}(X \times X),
$$

such that the $\Pi_{i}$ are mutually orthogonal idempotents and $\left(\Pi_{i}\right)_{*} H^{*}(X)=H^{i}(X)$.

(NB: "CK decomposition" is shorthand for "Chow-Künneth decomposition".)

Remark 2.5. The existence of a $C K$ decomposition for any smooth projective variety is part of Murre's conjectures [25], [18].

Definition 2.6 (Shen-Vial [35]). Let $X$ be a smooth projective variety of dimension $n$. Let $\Delta_{s m}^{X} \in A^{2 n}(X \times X \times X)$ be the class of the small diagonal

$$
\Delta_{s m}^{X}:=\{(x, x, x) \mid x \in X\} \subset X \times X \times X .
$$

An MCK decomposition is a CK decomposition $\left\{\Pi_{i}\right\}$ of $X$ that is multiplicative, i.e. it satisfies

$$
\Pi_{k} \circ \Delta_{s m}^{X} \circ\left(\Pi_{i} \times \Pi_{j}\right)=0 \text { in } A^{2 n}(X \times X \times X) \text { for all } i+j \neq k .
$$

(NB: "MCK decomposition" is shorthandfor "multiplicative Chow-Künneth decomposition".)

Remark 2.7. The small diagonal (seen as a correspondence from $X \times X$ to $X$ ) induces the multiplication morphism

$$
\Delta_{s m}^{X}: \quad h(X) \otimes h(X) \rightarrow h(X) \text { in } \mathcal{M}_{\text {rat }} .
$$

Suppose $X$ has a CK decomposition

$$
h(X)=\bigoplus_{i=0}^{2 n} h^{i}(X) \text { in } \mathcal{M}_{\text {rat }}
$$


By definition, this decomposition is multiplicative if for any $i, j$ the composition

$$
h^{i}(X) \otimes h^{j}(X) \rightarrow h(X) \otimes h(X) \stackrel{\Delta_{s m}^{X}}{\longrightarrow} h(X) \text { in } \mathcal{M}_{\text {rat }}
$$

factors through $h^{i+j}(X)$. It follows that if $X$ has an MCK decomposition, then setting

$$
A_{(j)}^{i}(X):=\left(\Pi_{2 i-j}^{X}\right)_{*} A^{i}(X),
$$

one obtains a bigraded ring structure on the Chow ring: that is, the intersection product sends $A_{(j)}^{i}(X) \otimes A_{\left(j^{\prime}\right)}^{i^{\prime}}(X)$ to $A_{\left(j+j^{\prime}\right)}^{i+i^{\prime}}(X)$.

The property of having an MCK decomposition is severely restrictive, and is closely related to Beauville's "weak splitting property" [3]. For more ample discussion, and examples of varieties with an MCK decomposition, we refer to [35, Section 8] and [39] and [36].

Lemma 2.8 (Vial [39]). Let $X, X^{\prime}$ be birational hyperkähler varieties. Then $X$ has an MCK decomposition if and only if $X^{\prime}$ has one.

Proof. This is noted in [39, Introduction]; the idea (as indicated in loc. cit.) is that Rieß's result [33] implies that $X$ and $X^{\prime}$ have isomorphic Chow motives and the isomorphism is compatible with the multiplicative structure.

More precisely: let $\gamma: X \rightarrow X^{\prime}$ be a birational map between hyperkähler varieties of dimension $n$, and suppose $\left\{\Pi_{i}^{X}\right\}$ is an MCK decomposition for $X$. Let $\Delta_{s m}^{X}, \Delta_{s m}^{X^{\prime}}$ denote the small diagonal of $X$ resp. $X^{\prime}$. As explained in [35, Section 6], the argument of [33] gives the equality

$$
\Gamma_{\gamma} \circ \Delta_{s m}^{X} \circ{ }^{t} \Gamma_{\gamma \times \gamma}=\Delta_{s m}^{X^{\prime}} \text { in } A^{2 n}\left(X^{\prime} \times X^{\prime} \times X^{\prime}\right) .
$$

The prescription

$$
\Pi_{i}^{X^{\prime}}:=\Gamma_{\gamma} \circ \pi_{i}^{X} \circ{ }^{t} \Gamma_{\gamma} \in A^{n}\left(X^{\prime} \times X^{\prime}\right)
$$

defines a CK decomposition for $F^{\prime}$. (The $\Pi_{i}^{X^{\prime}}$ are orthogonal idempotents thanks to Rieß's result that $\Gamma_{\gamma} \circ{ }^{t} \Gamma_{\gamma}=\Delta_{X^{\prime}}$ and ${ }^{t} \Gamma_{\gamma} \circ \Gamma_{\gamma}=\Delta_{X}[33]$.)

To see this CK decomposition $\left\{\Pi_{i}^{X^{\prime}}\right\}$ is multiplicative, let us consider integers $i, j, k$ such that $i+j \neq k$. It follows from the above equalities that

$$
\begin{aligned}
\Pi_{k}^{X^{\prime}} \circ \Delta_{s m}^{X^{\prime}} \circ\left(\Pi_{i}^{X^{\prime}} \times \Pi_{j}^{X^{\prime}}\right) & =\Gamma_{\gamma} \circ \Pi_{k}^{X} \circ{ }^{t} \Gamma_{\gamma} \circ \Gamma_{\gamma} \circ \Delta_{s m}^{X} \circ{ }^{t} \Gamma_{\gamma \times \gamma} \circ \Gamma_{\gamma \times \gamma} \circ\left(\Pi_{i}^{X} \times \Pi_{j}^{X}\right) \circ{ }^{t} \Gamma_{\gamma} \\
& =\Gamma_{\gamma} \circ \Pi_{k}^{X} \circ \Delta_{s m}^{X} \circ\left(\Pi_{i}^{X} \times \Pi_{j}^{X}\right) \circ{ }^{t} \Gamma_{\gamma} \\
& =0 \text { in } A^{2 n}\left(X^{\prime} \times X^{\prime}\right) .
\end{aligned}
$$

(Here we have again used Rieß's result that $\Gamma_{\gamma} \circ{ }^{t} \Gamma_{\gamma}=\Delta_{X^{\prime}}$ and ${ }^{t} \Gamma_{\gamma} \circ \Gamma_{\gamma}=\Delta_{X}$.)

2.3. MCK for $K 3^{[2]}$.

Theorem 2.9 (Shen-Vial [35]). Let $S$ be a $K 3$ surface, and $X=S^{[2]}$. There exists an MCK decomposition $\left\{\Pi_{j}^{X}\right\}$ for $X$. In particular, setting

$$
A_{(j)}^{i}(X):=\left(\Pi_{2 i-j}^{X}\right)_{*} A^{i}(X)
$$

defines a bigraded ring structure $A_{(*)}^{*}(X)$ on $A^{*}(X)$. Moreover, $A_{(*)}^{*}(X)$ coincides with the bigrading defined by the Fourier transform. 
Proof. The existence of $\left\{\Pi_{j}^{X}\right\}$ is a special case of [35, Theorem 13.4]. The "moreover" part is [35, Theorem 15.8].

Remark 2.10. The first statement of theorem 2.9 actually holds for $X=S^{[r]}$ for any $r \in \mathbb{N}$ [39].

Any $K 3$ surface $S$ has an MCK decomposition [35]. Since this property is stable under products, $S^{2}$ has an MCK decomposition. The following lemma records a basic compatibility between the bigradings on $A^{*}\left(S^{[2]}\right)$ and on $A^{*}\left(S^{2}\right)$ :

Lemma 2.11. Let $S$ be a $K 3$ surface, and $X=S^{[2]}$. Let $\Psi \in A^{4}\left(X \times S^{2}\right)$ be the correspondence coming from the diagram

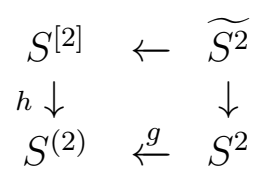

(the arrow labelled $h$ is the Hilbert-Chow morphism; the right vertical arrow is the blow-up of the diagonal). Then

$$
\begin{aligned}
& (\Psi)_{*} R(X) \subset R\left(S^{2}\right), \\
& \left({ }^{t} \Psi\right)_{*} R\left(S^{2}\right) \subset R(X),
\end{aligned}
$$

where $R=A_{(4)}^{4}$ or $A_{(2)}^{4}$ or $A_{(2)}^{2}$ or $A_{(0)}^{2} \cap A_{h o m}^{2}$.

Proof. We prove the statement for ${ }^{t} \Psi$ and $R=A_{(2)}^{2}$ or $A_{(0)}^{2} \cap A_{\text {hom }}^{2}$, which are the only cases we'll be using (the other statements can be proven similarly). By construction of the MCK decomposition for $X$, there is a relation

$$
\Pi_{k}^{X}=\frac{1}{2}{ }^{t} \Psi \circ \Pi_{k}^{S^{2}} \circ \Psi+\text { Rest } \text { in } A^{4}(X \times X), \quad(k=0,2,4,6,8),
$$

where $\left\{\Pi_{k}^{S^{2}}\right\}$ is a product MCK decomposition for $S^{2}$, and "Rest" is a term coming from $\Delta_{S} \subset$ $S \times S$ which does not act on $A^{4}(X)$ and on $A_{A J}^{2}(X)$. Since $\frac{1}{2}^{t} \Psi \circ \Psi$ is the identity on $A_{\text {hom }}^{2}(X)=$ $A_{A J}^{2}(X)$, we can write

$$
\left({ }^{t} \Psi\right)_{*}\left(\Pi_{k}^{S^{2}}\right)_{*}=\left({ }^{t} \Psi \circ \Pi_{k}^{S^{2}}\right)_{*}=\left(\frac{1}{2}{ }^{t} \Psi \circ \Psi \circ{ }^{t} \Psi \circ \Pi_{k}^{S^{2}}\right)_{*}: \quad A_{\text {hom }}^{2}\left(S^{2}\right) \rightarrow A_{\text {hom }}^{2}(X) .
$$

In view of sublemma 2.12 below, this implies

$$
\left({ }^{t} \Psi\right)_{*}\left(\Pi_{k}^{S^{2}}\right)_{*}=\left(\frac{1}{2}{ }^{t} \Psi \circ \Pi_{k}^{S^{2}} \circ \Psi \circ{ }^{t} \Psi\right)_{*}: \quad A_{h o m}^{2}\left(S^{2}\right) \rightarrow A_{h o m}^{2}(X) .
$$

But then, plugging in relation (3), we find

$$
\left({ }^{t} \Psi\right)_{*}\left(\Pi_{k}^{S^{2}}\right)_{*} A_{h o m}^{2}\left(S^{2}\right) \subset\left(\Pi_{k}^{X}\right)_{*} A_{h o m}^{2}(X) .
$$

Taking $k=2$, this proves

Taking $k=4$, this proves

$$
\left({ }^{t} \Psi\right)_{*} A_{(2)}^{2}\left(S^{2}\right) \subset A_{(2)}^{2}(X)
$$

$$
\left({ }^{t} \Psi\right)_{*}\left(A_{(0)}^{2}\left(S^{2}\right) \cap A_{\text {hom }}^{2}\left(S^{2}\right)\right) \subset A_{(0)}^{2}(X) \cap A_{\text {hom }}^{2}(X) .
$$


Sublemma 2.12. There is commutativity

$$
\Psi \circ{ }^{t} \Psi \circ \Pi_{k}^{S^{2}}=\Pi_{k}^{S^{2}} \circ \Psi \circ{ }^{t} \Psi \text { in } A^{4}\left(S^{4}\right) .
$$

To prove the sublemma, we remark that $h_{*} h^{*}=2 \mathrm{id}: A^{i}\left(S^{(2)}\right) \rightarrow A^{i}\left(S^{(2)}\right)$, and so

$$
\left(\Psi \circ{ }^{t} \Psi\right)_{*}=2 g^{*} g_{*}=2\left(\Delta_{S^{2}}+\Gamma_{\iota}\right)_{*}: \quad A^{i}\left(S^{2}\right) \rightarrow A^{i}\left(S^{2}\right),
$$

where $\iota$ denotes the involution switching the two factors. But $\left\{\Pi_{k}^{S^{2}}\right\}$, being a product decomposition, is symmetric and hence

$$
\Gamma_{\iota} \circ \Pi_{k}^{S^{2}} \circ \Gamma_{\iota}=(\iota \times \iota)^{*} \Pi_{k}^{S^{2}}=\Pi_{k}^{S^{2}} \text { in } A^{4}\left(S^{4}\right) .
$$

This implies commutativity

$$
\Gamma_{\iota} \circ \Pi_{k}^{S^{2}}=\Pi_{k}^{S^{2}} \circ \Gamma_{\iota} \text { in } A^{4}\left(S^{4}\right),
$$

which proves the sublemma.

Remark 2.13. Lemma 2.11 is probably true for any $(i, j)$ (i.e., $\Psi$ should be "of pure grade 0" in the language of [36, Definition 1.1]). I have not been able to prove this.

\subsection{MCK for $S \times S$.}

Notation 2.14. Let $\mathcal{S} \rightarrow B$ be a family (i.e., a smooth projective morphism). For $r \in \mathbb{N}$, we write $\mathcal{S}^{r / B}$ for the relative $r$-fold fibre product

$$
\mathcal{S}^{r / B}:=\mathcal{S} \times_{B} \mathcal{S} \times_{B} \cdots \times_{B} \mathcal{S}
$$

(r copies of $\mathcal{S})$.

Proposition 2.15. Let $\mathcal{S} \rightarrow B$ be a family of $K 3$ surfaces. There exist relative correspondences

$$
\Pi_{j}^{\mathcal{S}^{2 / B}} \in A^{4}\left(\mathcal{S}^{4 / B}\right)(j=0,2,4,6,8),
$$

such that

(i) for each $b \in B$, the restriction

$$
\Pi_{j}^{\left(S_{b}\right)^{2}}:=\left.\Pi_{j}^{\mathcal{S}^{2 / B}}\right|_{\left(S_{b}\right)^{4}} \in A^{4}\left(\left(S_{b}\right)^{4}\right)
$$

defines a self-dual MCK decomposition for $\left(S_{b}\right)^{2}$;

(ii) there is a decomposition

$$
\Pi_{2}^{\mathcal{S}^{2 / B}}=P_{1} \circ Q_{1}+P_{2} \circ Q_{2} \text { in } A^{4}\left(\mathcal{S}^{4 / B}\right),
$$

where $P_{i} \in A^{2}\left(\mathcal{S}^{4 / B}\right)$ and $Q_{i} \in A^{6}\left(\mathcal{S}^{4 / B}\right)$ for $i=1,2$.

Proof. (i) On any $K 3$ surface $S_{b}$, there is the distinguished 0-cycle $\mathfrak{o}_{S_{b}}$ such that $c_{2}\left(S_{b}\right)=24 \mathfrak{o}_{S_{b}}$ [4]. Let $p_{i}: \mathcal{S} \times{ }_{B} \mathcal{S} \rightarrow \mathcal{S}, i=1,2$, denote the projections to the two factors. Let $T_{\mathcal{S} / B}$ denote the relative tangent bundle. The assignment

$$
\begin{aligned}
& \Pi_{0}^{\mathcal{S}}:=\left(p_{1}\right)^{*}\left(\frac{1}{24} c_{2}\left(T_{\mathcal{S} / B}\right)\right) \quad A^{2}\left(\mathcal{S} \times{ }_{B} \mathcal{S}\right), \\
& \Pi_{4}^{\mathcal{S}}:=\left(p_{2}\right)^{*}\left(\frac{1}{24} c_{2}\left(T_{\mathcal{S} / B}\right)\right) \quad A^{2}\left(\mathcal{S} \times{ }_{B} \mathcal{S}\right), \\
& \Pi_{2}^{\mathcal{S}}:=\Delta_{\mathcal{S}}-\Pi_{0}^{\mathcal{S}}-\Pi_{4}^{\mathcal{S}}
\end{aligned}
$$


defines (by restriction) an MCK decomposition for each fibre:

$$
\Pi_{j}^{S_{b}}:=\left.\Pi_{j}^{\mathcal{S}}\right|_{S_{b} \times S_{b}} \quad \in A^{2}\left(S_{b} \times S_{b}\right) \quad(j=0,2,4)
$$

is an MCK decomposition [35, Example 8.17].

Next, we consider the fourfold relative fibre product $\mathcal{S}^{4 / B}$. Let

$$
p_{i j}: \mathcal{S}^{4 / B} \rightarrow \mathcal{S}^{2 / B} \quad(1 \leq i<j \leq 4)
$$

denote projection to the $i$-th and $j$-th factor. We set

$$
\Pi_{j}^{\mathcal{S}^{2 / B}}:=\sum_{k+\ell=j}\left(p_{13}\right)^{*}\left(\Pi_{k}^{\mathcal{S}}\right) \cdot\left(p_{24}\right)^{*}\left(\Pi_{\ell}^{\mathcal{S}}\right) \in A^{4}\left(\mathcal{S}^{4 / B}\right), \quad(j=0,2,4,6,8) .
$$

By construction, the restriction to each fibre induces an MCK decomposition (the "product MCK decomposition")

$$
\Pi_{j}^{\left(S_{b}\right)^{2}}:=\left.\Pi_{j}^{\mathcal{S}^{2 / B}}\right|_{\left(S_{b}\right)^{4}}=\sum_{k+\ell=j} \Pi_{k}^{S_{b}} \times \Pi_{\ell}^{S_{b}} \in A^{4}\left(\left(S_{b}\right)^{4}\right), \quad(j=0,2,4,6,8) .
$$

(ii) Define

$$
\begin{aligned}
P_{1} & :=\left(p_{13}\right)^{*}\left(\Pi_{2}^{\mathcal{S}}\right) \quad \in A^{2}\left(\mathcal{S}^{4 / B}\right), \\
Q_{1} & :=\left(p_{134}\right)^{*}\left(\Delta_{\mathcal{S}, s m}\right) \cdot\left(p_{2}\right)^{*}\left(\frac{1}{24} c_{2}\left(T_{\mathcal{S} / B}\right)\right) \in A^{6}\left(\mathcal{S}^{4 / B}\right), \\
P_{2} & :=\left(p_{24}\right)^{*}\left(\Pi_{2}^{\mathcal{S}}\right) \quad \in A^{2}\left(\mathcal{S}^{4 / B}\right), \\
Q_{2} & :=\left(p_{234}\right)^{*}\left(\Delta_{\mathcal{S}, s m}\right) \cdot\left(p_{1}\right)^{*}\left(\frac{1}{24} c_{2}\left(T_{\mathcal{S} / B}\right)\right) \in A^{6}\left(\mathcal{S}^{4 / B}\right) .
\end{aligned}
$$

Here $p_{134}: \mathcal{S}^{4 / B} \rightarrow \mathcal{S}^{3 / B}$ is projection on the first, third and fourth factor (and similarly for $p_{2}$, etc.), and $\Delta_{\mathcal{S}, s m}$ is the "relative small diagonal" (i.e., the image of the natural morphism $\mathcal{S} \rightarrow \mathcal{S}^{3 / B}$.

We will now show that for each $b \in B$, there is equality

$$
\begin{aligned}
& \left.\left(P_{1} \circ Q_{1}\right)\right|_{\left(S_{b}\right)^{4}}=\Pi_{2}^{S_{b}} \times \Pi_{0}^{S_{b}} \in A^{4}\left(\left(S_{b}\right)^{4}\right), \\
& \left.\left(P_{2} \circ Q_{2}\right)\right|_{\left(S_{b}\right)^{4}}=\Pi_{0}^{S_{b}} \times \Pi_{2}^{S_{b}} \in A^{4}\left(\left(S_{b}\right)^{4}\right) .
\end{aligned}
$$

This suffices to prove the proposition, because it implies that $P_{1} \circ Q_{1}+P_{2} \circ Q_{2}$ restricts to

$$
\Pi_{2}^{\left(S_{b}\right)^{2}}=\Pi_{2}^{S_{b}} \times \Pi_{0}^{S_{b}}+\Pi_{0}^{S_{b}} \times \Pi_{2}^{S_{b}} \in A^{4}\left(\left(S_{b}\right)^{4}\right),
$$

which is part of a product MCK decomposition on each fibre. 
For a given $S_{b}$ let $x=\mathfrak{o}_{S_{b}} \in A^{2}\left(S_{b}\right)$ denote the distinguished 0-cycle of [4]. We note that

$$
\begin{aligned}
\left.\left(P_{1} \circ Q_{1}\right)\right|_{\left(S_{b}\right)^{4}} & =\left(P _ { 1 } | _ { ( S _ { b } ) ^ { 4 } } \circ \left(\left.Q_{1}\right|_{\left(S_{b}\right)^{4}}\right.\right. \\
& =\left(\left(p_{13}\right)^{*}\left(\Pi_{2}^{S_{b}}\right)\right) \circ\left(\left\{(s, x, s, s) \in\left(S_{b}\right)^{4}\right\}\right) \\
& =\left(p_{1256}\right)_{*}\left(\left(p_{35}\right)^{*}\left(\Pi_{2}^{S_{b}}\right) \cdot\left(\{(s, x, s, s)\} \times S_{b} \times S_{b}\right)\right) \\
& =\left(p_{1256}\right)_{*}\left(\left(p_{15}\right)^{*}\left(\Pi_{2}^{S_{b}}\right) \cdot\left(\{(s, x, s, s)\} \times S_{b} \times S_{b}\right)\right) \\
& =\left(p_{13}\right)^{*}\left(\Pi_{2}^{S_{b}}\right) \cdot\left(\{(s, x)\} \times S_{b} \times S_{b}\right) \text { in } A^{4}\left(\left(S_{b}\right)^{4}\right) \\
& =\Pi_{2}^{S_{b}} \times x \times S_{b} \text { in } A^{4}\left(\left(S_{b}\right)^{4}\right) .
\end{aligned}
$$

Likewise,

$$
\begin{aligned}
\left.\left(P_{2} \circ Q_{2}\right)\right|_{\left(S_{b}\right)^{4}} & =\left(\left.P_{2}\right|_{\left(S_{b}\right)^{4}} \circ\left(\left.Q_{2}\right|_{\left(S_{b}\right)^{4}}\right)\right. \\
& =\left(\left(p_{24}\right)^{*}\left(\Pi_{2}^{S_{b}}\right)\right) \circ\left(\left\{(x, s, s, s) \in\left(S_{b}\right)^{4}\right\}\right) \\
& =\left(p_{1256}\right)_{*}\left(\left(p_{46}\right)^{*}\left(\Pi_{2}^{S_{b}}\right) \cdot\left(\{(x, s, s, s)\} \times S_{b} \times S_{b}\right)\right) \\
& =\left(p_{1256}\right)_{*}\left(\left(p_{26}\right)^{*}\left(\Pi_{2}^{S_{b}}\right) \cdot\left(\{(x, s, s, s)\} \times S_{b} \times S_{b}\right)\right) \\
& =\left(p_{24}\right)^{*}\left(\Pi_{2}^{S_{b}}\right) \cdot\left(\{(x, s)\} \times S_{b} \times S_{b}\right) \text { in } A^{4}\left(\left(S_{b}\right)^{4}\right) \\
& =x \times S_{b} \times \Pi_{2}^{S_{b}} \text { in } A^{4}\left(\left(S_{b}\right)^{4}\right) .
\end{aligned}
$$

This proves the equalities (4), and so the proposition is proven.

\subsection{Relative MCK for $K 3^{[2]}$.}

Proposition 2.16. Let $\mathcal{S} \rightarrow B$ be a family of $K 3$ surfaces (i.e. each fibre $S_{b}$ is a $K 3$ surface), and let $\mathcal{X} \rightarrow B$ be the family of associated Hilbert schemes (i.e., a fibre $X_{b}$ is $\left(S_{b}\right)^{[2]}$ ). There exist relative correspondences

$$
\Pi_{j}^{\mathcal{X}} \in A^{4}\left(\mathcal{X} \times_{B} \mathcal{X}\right)(j=0,2,4,6,8),
$$

such that for each $b \in B$, the restrictions

$$
\Pi_{j}^{X_{b}}:=\left.\Pi_{j}^{\mathcal{X}}\right|_{X_{b} \times X_{b}} \in A^{4}\left(X_{b} \times X_{b}\right) \quad(j=0,2,4,6,8)
$$

define an MCK decomposition for $X_{b}$.

Proof. The construction of an MCK decomposition for $X_{b}$ given in [35, Theorem 13.4] can be done in a relative setting. That is, let $\left\{\Pi_{j}^{\mathcal{S}}\right\}$ be a relative MCK decomposition for $\mathcal{S}$ as in proposition 2.15, and let $\left\{\Pi_{j}^{\mathcal{S}^{2 / B}}\right\}$ be the induced relative MCK decomposition for $\mathcal{S}^{2 / B}$ as in proposition 2.15. Let

$$
\mathcal{Z} \rightarrow B
$$


be the family obtained by blowing-up $\mathcal{S} \times{ }_{B} \mathcal{S}$ along the relative diagonal $\Delta_{\mathcal{S}}$. As in the proof of [35, Propositions 13.2 and 13.3 $]^{1}$, one can use $\left\{\Pi_{j}^{\mathcal{S}^{2 / B}}\right\}$ and $\left\{\Pi_{j}^{\mathcal{S}}\right\}$ to define relative correspondences

$$
\Pi_{j}^{\mathcal{Z}} \in A^{4}\left(\mathcal{Z} \times{ }_{B} \mathcal{Z}\right) \quad(j=0,2,4,6,8),
$$

which restrict to an MCK decomposition of each fibre $Z_{b}$. Let

$$
p: \mathcal{Z} \rightarrow \mathcal{X}
$$

denote the morphism of $B$-schemes induced by the action of the symmetric group $\mathfrak{S}_{2}$, and let $\Gamma_{p} \in A^{4}\left(\mathcal{Z} \times_{B} \mathcal{X}\right)$ be the graph of $p$. We define

$$
\Pi_{j}^{\mathcal{X}}:=\frac{1}{2} \Gamma_{p} \circ \Pi_{j}^{\mathcal{Z}} \circ{ }^{t} \Gamma_{p} \quad \in A^{4}\left(\mathcal{X} \times_{B} \mathcal{X}\right) \quad(j=0,2,4,6,8) .
$$

The restrictions $\Pi_{j}^{X_{b}}:=\left.\Pi_{j}^{\mathcal{X}}\right|_{X_{b} \times X_{b}}$ define an MCK decomposition for each fibre by [35, Theorem 13.4].

\subsection{Multiplicative structure of Chow ring of $K 3^{[2]}$.}

Theorem 2.17 (Shen-Vial [35]). Let $S$ be a K3 surface, and $X=S^{[2]}$.

(i) Intersection product induces a surjection

$$
A_{(2)}^{2}(X) \otimes A_{(2)}^{2}(X) \rightarrow A_{(4)}^{4}(X) .
$$

(ii) There is a distinguished class $l \in A_{(0)}^{2}(X)$ such that intersection induces an isomorphism

$$
\cdot l: \quad A_{(2)}^{2}(X) \stackrel{\cong}{\rightarrow} A_{(2)}^{4}(X) .
$$

Proof. This is [35, Theorem 3].

\subsection{Refined CK decomposition.}

Theorem 2.18 (Vial [38]). Let $X$ be a smooth projective variety of dimension $n \leq 5$. Assume the Lefschetz standard conjecture $B(X)$ holds (in particular, the Künneth components $\pi_{i} \in$ $H^{2 n}(X \times X)$ are algebraic). Then there is a splitting into mutually orthogonal idempotents

$$
\pi_{i}=\sum_{j} \pi_{i, j} \in H^{2 n}(X \times X),
$$

such that

$$
\left(\pi_{i, j}\right)_{*} H^{*}(X)=g r_{\widetilde{N}}^{j} H^{i}(X)
$$

In particular,

$$
\begin{aligned}
& \left(\pi_{2,1}\right)_{*} H^{j}(X)=H^{2}(X) \cap F^{1}, \\
& \left(\pi_{2,0}\right)_{*} H^{j}(X)=H_{t r}^{2}(X) .
\end{aligned}
$$

\footnotetext{
${ }^{1}$ The statement and proof of [35, Proposition 13.2] should be slightly modified, as noted in [36, Remark 2.8].
} 
(Here $F^{*}$ denotes the Hodge filtration, and $H_{t r}^{2}(X)$ is the orthogonal complement to $H^{2}(X) \cap F^{1}$ under the pairing

$$
\begin{aligned}
H^{2}(X) \otimes H^{2}(X) & \rightarrow \mathbb{Q}, \\
a \otimes b & \left.\mapsto a \cup h^{n-2} \cup b .\right)
\end{aligned}
$$

The projector $\pi_{2,1}$ is supported on $C \times D$, where $C \subset X$ is a curve and $D \subset X$ is a divisor.

Proof. This is [38, Theorem 1].

\subsection{The automorphism group of $K 3^{[2]}$.}

Proposition 2.19 (Boissière et alii [8]). Let $S$ be a projective K3 surface of Picard number $\rho(S)=1$, and let $X=S^{[2]}$. Suppose $\operatorname{Pic}(S)$ is generated by a divisor $H$ with $H^{2}=2$. Then $\operatorname{Aut}(X)=\mathbb{Z} / 2 \mathbb{Z}$, and the non-trivial involution ८ of $X$ is anti-symplectic, induced by the covering involution of $S$.

Proof. This is [8, Proposition 5.1].

Theorem 2.20 (Boissière et alii [8]). Let $S$ be a projective K3 surface of Picard number $\rho(S)=$ 1. Suppose $\operatorname{Pic}(S)$ is generated by a divisor $H$ with $H^{2}=2 t, t \geq 2$.

(i) The Hilbert scheme $X=S^{[2]}$ has a non-trivial automorphism if and only if there exists an ample divisor $D \in \operatorname{Pic}(X)$ of square 2 (with respect to the Beauville-Bogomolov quadratic form). This is the case for $t=1,10,13,17, \ldots$

(ii) If $\operatorname{Aut}(X) \neq 0$ then $\operatorname{Aut}(X)=\mathbb{Z} / 2 \mathbb{Z}$ and the only non-trivial automorphism is an antisymplectic involution $\iota$ leaving the divisor $D$ invariant (i.e. $\iota^{*}(D)=D$ in $N S(X)$ ).

Proof. Statement (i) is [8, Theorem 5.5] (combined with results concerning solutions of Pell's equation to compute the first values of $t$; these values are stated in [8, Introduction]). Statement (ii) is [8, Lemma 5.3].

Proposition 2.21 (Beauville [1]). Let $S \subset \mathbb{P}^{3}$ be a smooth quartic with Picard number $\rho(S)=1$, and let $X=S^{[2]}$. Let $G$ denote the Grassmannian of lines in $\mathbb{P}^{3}$, and let $\phi: X \rightarrow G$ be the morphism sending a length-two subscheme $Z$ to its one-dimensional span $<Z>\subset \mathbb{P}^{3}$.

(i) There exists an anti-symplectic involution

$$
\iota: \quad X \rightarrow X
$$

defined by sending $Z \in X$ to the residual subscheme of $\langle Z>\cap S$, i.e.

$$
<Z>\cap S=Z \amalg \iota(Z) .
$$

(ii) There exists an ample divisor $D \in A^{1}(X)$ of square 2 (with respect to the BeauvilleBogomolov form), and such that the linear system $|D|$ is base-point-free. Define the morphism $f$ as the composition

$$
f: X \stackrel{\phi}{\rightarrow} G \stackrel{\psi}{\rightarrow} \mathbb{P}^{5},
$$

where $\psi$ is the Plücker embedding. Then $f$ is the same as the morphism defined by $|D|$. 
(iii) The involution $\iota$ acts on $N S(X)$ as reflection in the span of $D$.

(iv) The involution $\iota$ is non-natural (i.e., there exists no pair $\left(S^{\prime}, \tau\right)$ with $S^{\prime}$ a $K 3$ surface and $\tau \in \operatorname{Aut}\left(S^{\prime}\right)$ and such that $\left.(X, \iota)=\left(\left(S^{\prime}\right)^{[2]}, \tau^{[2]}\right)\right)$.

Proof. Statement (i) is [1, Section 6]. Statements (ii) and (iii) are contained in [28, Section 4.1.2], or [8, Section 6.1]. Finally, point (iv) is proven in [9, page 6] by computing the index $\lambda(\iota$ ) (as defined in loc. cit.) of $\iota$.

Remark 2.22. Let $X$ be the Hilbert scheme $X=S^{[2]}$ of a generic quartic $S \subset \mathbb{P}^{3}$. Combining proposition 2.21 and theorem 2.20, it follows that Beauville's involution $\iota$ is the unique nontrivial automorphism of $X$.

Remark 2.23. Let $S \subset \mathbb{P}^{3}$ be a smooth quartic of any Picard number, and let $X=S^{[2]}$. Clearly, the above construction gives a rational map

$$
\iota: X \rightarrow X,
$$

which is well-defined outside of the locus of zero-dimensional subschemes contained in a line on $S$.

Remark 2.24. Oguiso [32, Section 4 Example 2] has used Beauville's involution to construct interesting automorphisms of $S^{[2]}$ where $S \subset \mathbb{P}^{3}$ is a certain quartic of Picard number 2. These automorphisms are non-natural and of positive entropy.

\subsection{EPW sextics.}

Definition 2.25 ([13]). Let $A \subset \wedge^{3} \mathbb{C}^{6}$ be a subspace which is Lagrangian with respect to the symplectic form on $\wedge^{3} \mathbb{C}^{6}$ given by the wedge product. The EPW sextic associated to $A$ is

$$
Y_{A}:=\left\{[v] \in \mathbb{P}\left(\mathbb{C}^{6}\right) \mid \operatorname{dim}\left(A \cap\left(v \wedge \wedge^{2} \mathbb{C}^{6}\right)\right) \geq 1\right\} \subset \mathbb{P}\left(\mathbb{C}^{6}\right)
$$

An EPW sextic is an $Y_{A}$ for some $A \subset \wedge^{3} \mathbb{C}^{6}$ Lagrangian.

Theorem 2.26 (O'Grady [29]). Let $X$ be a hyperkähler fourfold of $K 3^{[2]}-t y p e$. Assume there exists a divisor $D \in A^{1}(X)$ of Beauville-Bogomolov square 2 , and an anti-symplectic involution $\iota \in \operatorname{Aut}(X)$, such that there is a factorization

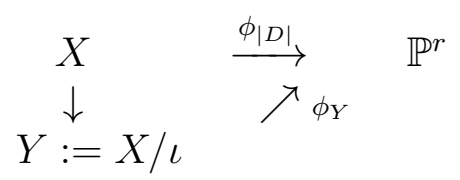

with $\phi_{Y}$ an embedding (here $\phi_{|D|}$ denotes the rational map associated to the linear system of $D$ ). Then $Y$ is an EPW sextic.

Proof. This is [29, Theorem 1.1(1)].

Corollary 2.27. Let $X$ be the Hilbert scheme $X=S^{[2]}$, where $S \subset \mathbb{P}^{3}$ is a smooth quartic with $\rho(S)=1$. Let $\iota \in \operatorname{Aut}(X)$ be the Beauville involution of [1]. Then $Y=X / \iota$ is an EPW sextic.

Proof. This is immediate from theorem 2.26 and proposition 2.21.

Remark 2.28. For more on EPW sextics and double EPW sextics, we refer the reader to [29], [31], [30]. 


\section{Degree $2 K 3$ SuRfaCeS}

As a warm-up before proving the main result (which is about the Hilbert square of $K 3$ surfaces of degree 4), we consider the Hilbert square of $K 3$ surfaces of degree 2 . This case is easy, because the anti-symplectic involution is natural.

Proposition 3.1. Let $S$ be a $K 3$ surface of degree 2 (i.e. there exists an ample divisor $H \in$ $\operatorname{Pic}(S)$ with $H^{2}=2$ ) and with $\rho(S)=1$. Let $X=S^{[2]}$, and let $\iota \in \operatorname{Aut}(X)$ be the unique non-trivial automorphism of proposition 2.19. Then

$$
\begin{aligned}
\iota^{*}=-\mathrm{id}: & A_{(2)}^{i}(X) \rightarrow A_{(2)}^{i}(X) \quad(i=2,4), \\
\iota^{*}=\mathrm{id}: & A_{(4)}^{4}(X) \rightarrow A_{(4)}^{4}(X) .
\end{aligned}
$$

Proof. The natural correspondence $\Psi \in A^{4}\left(X \times S^{2}\right)$ induces a a split injection

$$
\Psi_{*}: A^{4}(X) \rightarrow A^{4}\left(S^{2}\right),
$$

which is compatible with the bigrading $A_{(j)}^{4}$ for $j=2,4$ (lemma 2.11). The involution $\iota$ being natural, there is a commutative diagram

$$
\begin{array}{ccc}
A_{(j)}^{4}(X) & \stackrel{\Psi_{*}}{\longrightarrow} & A_{(j)}^{4}\left(S^{2}\right) \\
\downarrow \iota^{*} & & \downarrow(\iota \times \iota)^{*} \\
A^{4}(X) & \stackrel{\Psi_{*}}{\longrightarrow} & A^{4}\left(S^{2}\right)
\end{array}
$$

for $j=2,4$. We are thus reduced to proving a statement for $S^{2}$.

Lemma 3.2. Set-up as in proposition 3.1. Then

$$
A_{\text {hom }}^{2}(S)^{\iota}=0 \text {. }
$$

Proof. The quotient variety $S / \iota$ has geometric genus 0 . Since quotient singularities are rational singularities, there exists a resolution $Y \rightarrow S / \iota$ with $p_{g}(Y)=0$. Since $Y$ is not of general type, Bloch's conjecture is known to hold for $Y$ [6], i.e. $A_{\text {hom }}^{2}(Y)=0$. This implies that also $A_{\text {hom }}^{2}(S / \iota)=A_{\text {hom }}^{2}(S)^{\iota}=0$.

Lemma 3.2 implies that $\iota$ acts as - id on $A_{\text {hom }}^{2}(S)$. Looking at the action of $\iota$ on $H^{2,2}(S) \cong \mathbb{C}$, one finds that $\iota$ acts as id on $A_{(0)}^{2}(S)$. This implies that

$$
\begin{aligned}
& \iota^{*}=-\mathrm{id}: \quad A_{(2)}^{4}\left(S^{2}\right) \rightarrow A^{4}\left(S^{2}\right), \\
& \iota^{*}=\mathrm{id}: \quad A_{(2)}^{4}\left(S^{2}\right) \rightarrow A^{4}\left(S^{2}\right) .
\end{aligned}
$$

Using diagram (5), this proves proposition 3.1 for $A_{(2)}^{4}$ and for $A_{(4)}^{4}$.

It remains to prove the statement for $A_{(2)}^{2}(X)$. This can be done as follows: the above implies there is a decomposition

$$
A^{4}(X)=A^{4}(X)^{\iota} \oplus A_{(2)}^{4}(X)
$$

and so the correspondence

$$
\Gamma:=\Pi_{6}^{X} \circ\left(\Gamma_{\iota}+\Delta_{X}\right) \quad \in A^{4}(X \times X)
$$


acts trivially on $0-$ cycles:

$$
\Gamma_{*} A^{4}(X)=0 \text { in } A^{4}(X) .
$$

Using the Bloch-Srinivas argument [7], this implies $\Gamma$ is supported on $D \times X$, where $D \subset X$ is a divisor. This holds for any MCK decomposition $\left\{\Pi_{i}^{X}\right\}$ for $X$. Let us now take an MCK decomposition of $X$ that is self-dual (this exists: [36, Remark 2.8]). The transpose

$$
{ }^{t} \Gamma=\left(\Gamma_{\iota}+\Delta_{X}\right) \circ \Pi_{2}^{X} \in A^{4}(X \times X)
$$

is supported on $X \times D$. As such, it does not act on $A_{h o m}^{2}(X)=A_{A J}^{2}(X)$ :

$$
\left({ }^{t} \Gamma\right)_{*}=0: \quad A_{h o m}^{2}(X) \rightarrow A_{h o m}^{2}(X) .
$$

Since $A_{(2)}^{2}(X)=\left(\Pi_{2}^{X}\right)_{*} A_{\text {hom }}^{2}(X)$, this implies

$$
\left(\Gamma_{\iota}+\Delta_{X}\right)_{*}=0: A_{(2)}^{2}(X) \rightarrow A^{2}(X),
$$

proving the statement for $A_{(2)}^{2}(X)$.

\section{MAIN RESULT}

This section contains the proof of the main result of this note (theorem 4.1). The global strategy is as follows: we start by proving (theorem 4.2) that the involution $\iota$ has the expected action on $A_{(2)}^{2}(X)$. As will be apparent to the well-informed reader, the proof of theorem 4.2 is directly inspired by Voisin's seminal work on the Bloch/Hodge equivalence for complete intersections [42], [43], [45], reasoning family-wise and spreading out correspondences to the family. At the heart of our proof is a result of Voisin [42] concerning the triviality of certain Chow groups of the fourfold relative fibre product $\mathcal{S}^{4 / B}$ of the family of smooth quartic surfaces (theorem 4.5). Voisin's result is conditional to the standard conjectures; however, we manage to bypass the need for the standard conjectures by only using Voisin's result in codimension 2, where it is unconditional.

Next, we consider the action of the involution $\iota$ on 0 -cycles (theorem 4.15). Here, we rely on the result for $A_{(2)}^{2}(X)$, plus the relations in $A^{*}(X)$ discovered by Shen-Vial (theorem 2.17). In order to be able to use these relations, we apply once again (proposition 4.16) Voisin's method of "spread". This second application of the method of "spread" is easier than the first, as everything happens on $\mathcal{S} \times{ }_{B} \mathcal{S}$, rather than on the fourfold relative fibre product $\mathcal{S}^{4 / B}$.

Here is the main result of this note:

Theorem 4.1. Let $S \subset \mathbb{P}^{3}$ be a smooth quartic with Picard number $\rho(S)=1$, and let $X=S^{[2]}$. Let $\iota \in \operatorname{Aut}(X)$ be the non-symplectic involution of Beauville (cf. proposition 2.21). Then

$$
\begin{aligned}
\iota^{*}=-\mathrm{id}: & A_{(2)}^{i}(X) \rightarrow A_{(2)}^{i}(X) \text { for } i=2,4 ; \\
\iota^{*}=\mathrm{id}: & A_{(j)}^{4}(X) \rightarrow A_{(j)}^{4}(X) \text { for } j=0,4 .
\end{aligned}
$$

Theorem 4.1 is a combination of theorems 4.2 and 4.15 . 


\subsection{Action on $A_{(2)}^{2}$.}

Theorem 4.2. Let $X$ and $\iota$ be as in theorem 4.1. Then

$$
\iota^{*}=-\mathrm{id}: \quad A_{(2)}^{2}(X) \rightarrow A_{(2)}^{2}(X) .
$$

Proof. We consider the family

$$
\mathcal{S} \rightarrow B
$$

of all smooth quartics $S_{b}$ with Picard number $\rho\left(S_{b}\right)=1$. Here the base $B$ is a Zariski-open in a projective space $B \subset \bar{B}:=\mathbb{P} H^{0}\left(\mathbb{P}^{3}, \mathcal{O}_{\mathbb{P}^{3}}(4)\right)$. We will denote

$$
\mathcal{X} \rightarrow B
$$

the family of Hilbert schemes, and we write $X_{b}=\left(S_{b}\right)^{[2]}$ for a fibre of $\mathcal{X} \rightarrow B$. It will be convenient to also consider the family $\mathcal{S} \times{ }_{B} \mathcal{S}$ (whose fibres are products $S_{b} \times S_{b}$ ). This family is related to the family $\mathcal{X} \rightarrow B$ by a "hat" of morphisms over $B$

$$
\mathcal{X}^{\widehat{\widetilde{\mathcal{S} \times_{B} \mathcal{S}}} \searrow} \mathcal{S} \times_{B} \mathcal{S}
$$

where $\widetilde{\mathcal{S} \times{ }_{B} \mathcal{S}}$ is the blow-up of $\mathcal{S} \times{ }_{B} \mathcal{S}$ with centre the relative diagonal. This diagram (6) gives rise to relative correspondences

$$
\Psi \in A^{4}\left(\mathcal{X} \times{ }_{B} \mathcal{S} \times{ }_{B} \mathcal{S}\right), \quad{ }^{t} \Psi \in A^{4}\left(\mathcal{S} \times{ }_{B} \mathcal{S} \times{ }_{B} \mathcal{X}\right)
$$

(For details on relative correspondences, cf. [26], and also [12], [11], [27].) Restricting to a fibre over $b \in B$, diagram (6) induces the familiar diagram

$$
X_{b}=\left(S_{b}\right)^{[2]} \swarrow^{\widehat{S_{b} \times S_{b}}} \searrow S_{b} \times S_{b}
$$

(where $\widetilde{S_{b} \times S_{b}}$ is the blow-up of $S_{b} \times S_{b}$ along the diagonal), and the (absolute) correspondences

$$
\Psi_{b} \in A^{4}\left(X_{b} \times S_{b} \times S_{b}\right),{ }^{t} \Psi_{b} \in A^{4}\left(S_{b} \times S_{b} \times X_{b}\right) .
$$

The morphism $\phi_{b}: X_{b} \rightarrow G$ (where $G$ is the Grassmannian of lines in $\mathbb{P}^{3}$ ) extends to the family, in the sense that there is a morphism of $B$-schemes

$$
\phi: \mathcal{X} \rightarrow \mathcal{G}:=G \times B
$$

such that the restriction to a fibre gives $\phi_{b}$. This implies that the Beauville involution also extends to the family: there exists an involution of $B$-schemes

$$
\iota: \mathcal{X} \rightarrow \mathcal{X}
$$

such that restriction to a fibre gives the involution $\iota_{b}: X_{b} \rightarrow X_{b}$ of proposition 2.21.

Let $\Gamma_{\iota} \in A^{4}\left(\mathcal{X} \times_{B} \mathcal{X}\right)$ denote the graph of $\iota$. The fact that $\iota_{b}$ acts as -1 on $H^{2,0}\left(X_{b}\right)$ for all $b \in B$ implies that

$$
\left(\Gamma_{\iota_{b}}+\Delta_{X_{b}}\right) \circ\left(\pi_{2, t r}^{X_{b}}\right)=0 \quad \text { in } H^{8}\left(X_{b} \times X_{b}\right), \quad \forall b \in B .
$$


In view of the refined Chow-Künneth decomposition (theorem 2.18), this implies that

$$
\left(\Gamma_{\iota_{b}}+\Delta_{X_{b}}\right) \circ\left(\pi_{2}^{X_{b}}\right)=\gamma_{b} \quad \text { in } H^{8}\left(X_{b} \times X_{b}\right), \quad \forall b \in B
$$

where $\gamma_{b}$ is some cycle supported on $Y_{b} \times Y_{b}$, for $Y_{b} \subset X_{b}$ a divisor.

Let $\left\{\Pi_{j}^{\mathcal{X}}\right\}$ be a relative MCK decomposition as in proposition 2.16. The relation (7) implies the following: the relative correspondence

$$
\Gamma_{0}:=\left(\Gamma_{\iota}+\Delta_{\mathcal{X}}\right) \circ \Pi_{2}^{\mathcal{X}} \in A^{4}\left(\mathcal{X} \times_{B} \mathcal{X}\right)
$$

has the property that for each $b \in B$, there exists a divisor $Y_{b} \subset X_{b}$ and a cycle $\gamma_{b}$ supported on $Y_{b} \times Y_{b}$ such that

$$
\left.\left(\Gamma_{0}\right)\right|_{X_{b} \times X_{b}}=\gamma_{b} \text { in } H^{8}\left(X_{b} \times X_{b}\right) .
$$

At this point, we recall Voisin's "spread-out" result:

Proposition 4.3 (Voisin [42]). Let $\mathcal{X} \rightarrow B$ be a smooth projective morphism of relative dimension $n$. Let $\Gamma \in A^{n}\left(\mathcal{X} \times_{B} \mathcal{X}\right)$ be a cycle such that for all $b \in B$, there exists a closed algebraic subset $Y_{b} \subset X_{b}$ of codimension $c$, and a cycle $\gamma_{b} \in A_{n}\left(Y_{b} \times Y_{b}\right)$ such that

$$
\left.\Gamma\right|_{X_{b} \times X_{b}}=\gamma_{b} \text { in } H^{2 n}\left(X_{b} \times X_{b}\right) \text {. }
$$

Then there exists a closed algebraic subset $\mathcal{Y} \subset \mathcal{X}$ of codimension $c$, and a cycle $\gamma \in A_{*}\left(\mathcal{Y} \times{ }_{B} \mathcal{Y}\right)$ such that

$$
\left.\Gamma\right|_{X_{b} \times X_{b}}=\left.\gamma\right|_{X_{b} \times X_{b}} \text { in } H^{2 n}\left(X_{b} \times X_{b}\right) \quad \forall b \in B .
$$

Proof. This is a Hilbert schemes argument [42, Proposition 3.7].

Applying proposition 4.3 to $\Gamma_{0}$, it follows there exists a divisor $\mathcal{Y} \subset \mathcal{X}$ and a cycle $\gamma \in$ $A_{*}\left(\mathcal{Y} \times_{B} \mathcal{Y}\right)$ such that

$$
\left.\left(\Gamma_{0}-\gamma\right)\right|_{X_{b} \times X_{b}}=0 \quad \text { in } H^{8}\left(X_{b} \times X_{b}\right), \quad \forall b \in B .
$$

That is, the relative correspondence

$$
\Gamma_{1}:=\Gamma_{0}-\gamma \in A^{4}\left(\mathcal{X} \times_{B} \mathcal{X}\right)
$$

has the property of being homologically trivial on every fibre:

$$
\left.\left(\Gamma_{1}\right)\right|_{X_{b} \times X_{b}}=0 \quad \text { in } H^{8}\left(X_{b} \times X_{b}\right), \quad \forall b \in B .
$$

At this point, it is convenient to consider the family $\mathcal{S} \times{ }_{B} \mathcal{S}$ (of products of surfaces $S_{b} \times$ $S_{b}$ ), rather than the family $\mathcal{X}$ (of Hilbert schemes $\left(S_{b}\right)^{[2]}$ ). That is, we consider the relative correspondence

$$
\Gamma_{2}:=\Psi \circ \Gamma_{1} \circ{ }^{t} \Psi \quad \in A^{4}\left(\mathcal{S}^{4 / B}\right),
$$

where

$$
\mathcal{S}^{4 / B}:=\mathcal{S} \times{ }_{B} \mathcal{S} \times{ }_{B} \mathcal{S} \times{ }_{B} \mathcal{S}
$$

Since

$$
\left.\left(\Gamma_{2}\right)\right|_{\left(S_{b}\right)^{4}}=\left(\Psi_{b}\right) \circ\left(\left.\left(\Gamma_{1}\right)\right|_{X_{b} \times X_{b}}\right) \circ{ }^{t} \Psi_{b} \quad \text { in } A^{4}\left(\left(S_{b}\right)^{4}\right)
$$

(restriction and composition commute), the relative correspondence $\Gamma_{2}$ has the property of being homologically trivial on every fibre:

$$
\left.\left(\Gamma_{2}\right)\right|_{\left(S_{b}\right)^{4}}=0 \quad \text { in } H^{8}\left(\left(S_{b}\right)^{4}\right), \quad \forall b \in B .
$$


Thanks to the following result, we can improve this fibre-wise homological vanishing to a global homological vanishing:

Proposition 4.4 (Voisin [42]). Let $\Gamma \in A^{4}\left(\mathcal{S}^{4 / B}\right)$ be such that

$$
\left.\Gamma\right|_{\left(S_{b}\right)^{4}}=0 \text { in } H^{8}\left(\left(S_{b}\right)^{4}\right) \quad \forall b \in B .
$$

Then, after shrinking $B$ to a non-empty Zariski-open subset, we have

$$
\Gamma+\sum_{j=1}^{6} \psi_{j}=0 \text { in } H^{8}\left(\mathcal{S}^{4 / B}\right),
$$

where $\psi_{1}$ (resp. $\psi_{2}, \ldots, \psi_{6}$ ) is the restriction of a cycle on $B \times \mathbb{P}^{3} \times \mathbb{P}^{3} \times \mathcal{S} \times_{B} \mathcal{S}$ (resp. on a copy of $B \times \mathbb{P}^{3} \times \mathbb{P}^{3} \times \mathcal{S} \times{ }_{B} \mathcal{S}$, where the factors are permuted).

Proof. This is an extension of the Leray spectral sequence argument [42, Lemmas 3.11 and 3.12] to the fourfold relative fibre product $\mathcal{S}^{4 / B}$. The fact that such an extension is true is stated in [42, Proof of Theorem 4.10], which also uses the fourfold relative fibre product $\mathcal{S}^{4 / B}$.

Applying proposition 4.4 to $\Gamma_{2}$, we obtain a relative correspondence

$$
\Gamma_{3}:=\Gamma_{2}+\psi \quad \in A^{4}\left(\mathcal{S}^{4 / B}\right)
$$

that is homologically trivial (i.e. $\Gamma_{3} \in A_{h o m}^{4}\left(\mathcal{S}^{4 / B}\right)$. Here $\psi$ is a cycle of the form

$$
\psi=\psi_{1}+\cdots+\psi_{6} \quad \in A^{4}\left(\mathcal{S}^{4 / B}\right),
$$

where $\psi_{1}, \ldots, \psi_{6}$ are restrictions of cycles coming from larger varieties as in proposition 4.4.

We now come to the "trivial Chow groups" statement which is at the heart of our proof:

Theorem 4.5 (Voisin [42]). Let $\mathcal{S} \rightarrow B$ denote the family of all smooth hypersurfaces $S_{b} \subset \mathbb{P}^{3}$ of a given degree $d$ (where $d \geq 3$ ). Let

$$
i: \mathcal{S}_{0}^{4 / B} \subset \mathcal{S}^{4 / B}
$$

denote the complement of the small relative diagonal $\mathcal{S} \subset \mathcal{S}^{4 / B}$. There exists a smooth proper surjective morphism

$$
f: \widetilde{\mathcal{S}_{0}^{4 / B}} \rightarrow \mathcal{S}_{0}^{4 / B}
$$

and a smooth quasi-projective variety $M$ containing $\widetilde{\mathcal{S}_{0}^{4 / B}}$ as a Zariski-open and such that

$$
A_{\text {hom }}^{i}(M)=0 \quad \forall i \leq 4 \text {. }
$$

Proof. This is (contained in the proof of) [42, Proposition 4.11]. The variety $M$ is constructed as a projective bundle over the variety $\widehat{\left(\mathbb{P}^{3}\right)_{0}^{4}}$ of [42, Lemma 4.12].

The relative correspondence $\Gamma_{3}$ being homologically trivial, we also have that

$$
\Gamma_{4}:=f^{*} i^{*}\left(\Gamma_{3}\right) \quad \in A^{4}\left(\widetilde{\mathcal{S}_{0}^{4 / B}}\right)
$$

is homologically trivial. Now, if we assume the Lefschetz standard conjecture (or the Voisin standard conjecture [42, Conjecture 1.6], [45, Conjecture 2.29]) is true, we can find a cycle

$$
\bar{\Gamma}_{4} \in A^{4}(M)
$$


which restricts to $\Gamma_{4}$ and is homologically trivial. In view of theorem 4.5 , we then obtain a rational equivalence

$$
\bar{\Gamma}_{4}=0 \text { in } A^{4}(M),
$$

and we can conclude the argument. However, as we do not want to end up with a conditional statement we need to avoid recourse to the Voisin (or Lefschetz) standard conjecture. To this end, we slightly modify the relative correspondence $\Gamma_{3}$, by defining

$$
\Gamma_{5}:=\Gamma_{3} \circ \Pi_{2}^{\mathcal{S}^{2 / B}} \in A^{4}\left(\mathcal{S}^{4 / B}\right),
$$

where $\Pi_{2}^{\mathcal{S}^{2 / B}}$ is part of a "relative MCK decomposition" for $\mathcal{S}^{2 / B}$ as in proposition 2.15. Since $\Gamma_{3}$ is homologically trivial, $\Gamma_{5}$ is so as well:

$$
\Gamma_{5} \in A_{h o m}^{4}\left(\mathcal{S}^{4 / B}\right) .
$$

Now, using the factorization $\Pi_{2}^{\mathcal{S}^{2 / B}}=P_{1} \circ Q_{1}+P_{2} \circ Q_{2}$ of proposition 2.15, we obtain a factorization

$$
\Gamma_{5}=\Gamma_{3} \circ P_{1} \circ Q_{1}+\Gamma_{3} \circ P_{2} \circ Q_{2} \text { in } A^{4}\left(\mathcal{S}^{4 / B}\right),
$$

where $\Gamma_{3} \circ P_{j} \in A^{2}\left(\mathcal{S}^{4 / B}\right)$ and $Q_{j} \in A^{6}\left(\mathcal{S}^{4 / B}\right)$ for $j=1,2$. Moreover,

$$
\Gamma_{3} \circ P_{1}, \quad \Gamma_{3} \circ P_{2} \in A_{h o m}^{2}\left(\mathcal{S}^{4 / B}\right)
$$

(since $\Gamma_{3}$ is homologically trivial). It follows that the pullbacks

$$
\Gamma_{6, j}:=f^{*} i^{*}\left(\Gamma_{3} \circ P_{j}\right) \quad \in A^{2}\left(\widetilde{\mathcal{S}_{0}^{4 / B}}\right) \quad(j=1,2)
$$

are also homologically trivial. But the cycles $\Gamma_{6, j}$ can be extended to $M$, i.e. there exist

$$
\bar{\Gamma}_{6, j} \in A^{2}(M) \quad(j=1,2)
$$

which restrict to $\Gamma_{6, j}$ and are homologically trivial (indeed, the Voisin standard conjecture is true in codimension 2, essentially because the Hodge conjecture is true in codimension 1 [42]). But then, using theorem 4.5 , we find that

$$
\bar{\Gamma}_{6, j}=0 \text { in } A^{2}(M),
$$

and so

$$
\Gamma_{6, j}=0 \quad \text { in } A^{2}\left(\widetilde{\mathcal{S}_{0}^{4 / B}}\right) \quad(j=1,2) .
$$

It follows that also

$$
\Gamma_{3} \circ P_{j}=i_{*} f_{*} f^{*} i^{*}\left(\Gamma_{3} \circ P_{j}\right)=0 \quad \text { in } A^{2}\left(\mathcal{S}^{4 / B}\right) \quad(j=1,2)
$$

(note that $i_{*} i^{*}=\mathrm{id}$ on codimension 2 cycles, for dimension reasons), and so

$$
\Gamma_{5}=\Gamma_{3} \circ P_{1} \circ Q_{1}+\Gamma_{3} \circ P_{2} \circ Q_{2}=0 \text { in } A^{4}\left(\mathcal{S}^{4 / B}\right) .
$$

In particular, restricting to a fibre, we obtain

$$
\left.\left(\Gamma_{5}\right)\right|_{\left(S_{b}\right)^{4}}=0 \text { in } A^{4}\left(\left(S_{b}\right)^{4}\right) \quad \forall b \in B .
$$

We now make the connection with the relative correspondence $\Gamma_{0}$ that we started out with. 
Claim 4.6. We have

$$
\left(\left.\left(\Psi \circ \Gamma_{0} \circ{ }^{t} \Psi+\gamma^{\prime}+\psi^{\prime}\right)\right|_{\left(S_{b}\right)^{4}}\right)_{*}=0: \quad A_{A J}^{2}\left(S_{b} \times S_{b}\right) \rightarrow A_{A J}^{2}\left(S_{b} \times S_{b}\right) \quad \forall b \in B,
$$

where $\gamma^{\prime}$ is a cycle supported on $\mathcal{D} \times_{B} \mathcal{D}$ for some divisor $\mathcal{D} \subset \mathcal{S} \times_{B} \mathcal{S}$, and $\psi^{\prime}$ is a sum of restrictions of cycles coming from larger varieties as in proposition 4.4 .

Proof. Recall that $\Gamma_{0}$ was defined as

$$
\Gamma_{0}:=\left(\Gamma_{\iota}+\Delta_{\mathcal{X}}\right) \circ \Pi_{2}^{\mathcal{X}} \in A^{4}\left(\mathcal{X} \times_{B} \mathcal{X}\right),
$$

and $\Gamma_{1}$ was defined as the difference

$$
\Gamma_{1}:=\Gamma_{0}-\gamma \in A^{4}\left(\mathcal{X} \times_{B} \mathcal{X}\right)
$$

where $\gamma$ is a cycle supported on $\mathcal{Y} \times_{B} \mathcal{Y}$ for some divisor $\mathcal{Y} \subset \mathcal{X}$. The next step was to define

$$
\Gamma_{2}:=\Psi \circ \Gamma_{1} \circ{ }^{t} \Psi \quad \in A^{4}\left(\mathcal{S}^{4 / B}\right),
$$

and then

$$
\Gamma_{3}:=\Gamma_{2}+\psi \quad \in A^{4}\left(\mathcal{S}^{4 / B}\right),
$$

where $\psi$ is a sum of restrictions of cycles coming from larger varieties as in proposition 4.4. This implies that

$$
\Gamma_{3}=\Psi \circ \Gamma_{0} \circ{ }^{t} \Psi+\gamma_{1}+\psi \quad \in A^{4}\left(\mathcal{S}^{4 / B}\right),
$$

where $\gamma_{1}=\Psi \circ \gamma \circ{ }^{t} \Psi$ is supported on $\mathcal{D} \times_{B} \mathcal{D}$ for some divisor $\mathcal{D} \subset \mathcal{S} \times_{B} \mathcal{S}$. The relative correspondence $\Gamma_{5}$ was defined as $\Gamma_{5}:=\Gamma_{3} \circ \Pi_{2}^{\mathcal{S}^{2 / B}}$, and so (by substituting using equality (9)) we find an equality

$$
\Gamma_{5}=\Psi \circ \Gamma_{0} \circ{ }^{t} \Psi \circ \Pi_{2}^{\mathcal{S}^{2 / B}}+\gamma^{\prime}+\psi^{\prime} \in A^{4}\left(\mathcal{S}^{4 / B}\right),
$$

where $\gamma^{\prime}:=\gamma_{1} \circ \Pi_{2}^{\mathcal{S}^{2 / B}}$ is supported on $\mathcal{D} \times_{B} \mathcal{D}$, and $\psi^{\prime}:=\psi \circ \Pi_{2}^{\mathcal{S}^{2 / B}}$ is a sum of restrictions of cycles coming from larger varieties as in proposition 4.4. But we know that $\Gamma_{5}$ is rationally trivial on each fibre (equality (8)), and so equality (10) implies

$$
\left.\left(\Psi \circ \Gamma_{0} \circ{ }^{t} \Psi \circ \Pi_{2}^{\mathcal{S}^{2 / B}}\right)\right|_{\left(S_{b}\right)^{4}}+\left.\left(\gamma^{\prime}+\psi^{\prime}\right)\right|_{\left(S_{b}\right)^{4}}=0 \text { in } A^{4}\left(\left(S_{b}\right)^{4}\right) \quad \forall b \in B .
$$

Applying both sides of the equality of correspondences (11) to codimension 2 cycles implies claim 4.6, in view of the following lemma:

Lemma 4.7. For any $b \in B$, there is equality

$$
\left(\left.\left(\Psi \circ \Gamma_{0} \circ{ }^{t} \Psi \circ \Pi_{2}^{\mathcal{S}^{2 / B}}\right)\right|_{\left(S_{b}\right)^{4}}\right)_{*}=\left(\left.\left(\Psi \circ \Gamma_{0} \circ{ }^{t} \Psi\right)\right|_{\left(S_{b}\right)^{4}}\right)_{*}: \quad A_{A J}^{2}\left(S_{b} \times S_{b}\right) \rightarrow A_{A J}^{2}\left(S_{b} \times S_{b}\right) .
$$

Proof. We start by observing there is a commutativity relation

$$
\left(\Pi_{2}^{X_{b}} \circ{ }^{t} \Psi_{b}\right)_{*}=\left(\Pi_{2}^{X_{b}} \circ{ }^{t} \Psi_{b} \circ \Pi_{2}^{\left(S_{b}\right)^{2}}\right)_{*}: \quad A_{h o m}^{2}\left(S_{b} \times S_{b}\right) \rightarrow A_{h o m}^{2}\left(X_{b}\right) .
$$

Indeed, we have seen (lemma 2.11) that

$$
\begin{aligned}
& \left({ }^{t} \Psi\right)_{*} A_{(2)}^{2}\left(S_{b} \times S_{b}\right) \subset A_{(2)}^{2}\left(X_{b}\right), \\
& \left({ }^{t} \Psi_{b}\right)_{*}\left(A_{(0)}^{2}\left(S_{b} \times S_{b}\right) \cap A_{h o m}^{2}\left(S_{b} \times S_{b}\right)\right) \subset A_{(0)}^{2}\left(X_{b}\right) \cap A_{h o m}^{2}\left(X_{b}\right),
\end{aligned}
$$


and so

Since

$$
\left(\Pi_{2}^{X_{b}} \circ{ }^{t} \Psi_{b}\right)_{*}=0: \quad A_{(0)}^{2}\left(S_{b} \times S_{b}\right) \cap A_{h o m}^{2}\left(S_{b} \times S_{b}\right) \rightarrow A^{2}\left(X_{b}\right)
$$

it follows that

$$
A_{h o m}^{2}\left(S_{b} \times S_{b}\right)=A_{(2)}^{2}\left(S_{b} \times S_{b}\right) \oplus A_{(0)}^{2}\left(S_{b} \times S_{b}\right) \cap A_{h o m}^{2}\left(S_{b} \times S_{b}\right),
$$

$$
\begin{aligned}
& \operatorname{Im}\left(A_{\text {hom }}^{2}\left(S_{b} \times S_{b}\right) \stackrel{\left({ }^{t} \Psi_{b}\right)_{*}}{\longrightarrow} A^{2}\left(X_{b}\right) \stackrel{\left(\Pi_{2}^{X_{b}}\right)_{*}}{\longrightarrow} A_{(2)}^{2}\left(X_{b}\right)\right)= \\
& \operatorname{Im}\left(A_{(2)}^{2}\left(S_{b} \times S_{b}\right) \stackrel{\left({ }^{t} \Psi_{b}\right)_{*}}{\longrightarrow} A^{2}\left(X_{b}\right) \stackrel{\left(\Pi_{2}^{X_{b}}\right)_{*}}{\longrightarrow} A_{(2)}^{2}\left(X_{b}\right)\right)= \\
& \operatorname{Im}\left(A^{2}\left(S_{b} \times S_{b}\right) \stackrel{\left(\Pi_{2}^{\left(S_{b}\right)^{2}}\right)_{*}}{\longrightarrow} A_{(2)}^{2}\left(S_{b} \times S_{b}\right) \stackrel{\left({ }^{t} \Psi_{b}\right)_{*}}{\longrightarrow} A^{2}\left(X_{b}\right) \stackrel{\left(\Pi_{2}^{X_{b}}\right)_{*}}{\longrightarrow} A_{(2)}^{2}\left(X_{b}\right)\right) .
\end{aligned}
$$

This proves equality (12).

To prove lemma 4.7, one notes that the left-hand-side of lemma 4.7 is

$$
\left(\left.\Psi_{b} \circ\left(\Gamma_{0}\right)\right|_{\left(S_{b}\right)^{4}} \circ{ }^{t} \Psi_{b} \circ \Pi_{2}^{\left(S_{b}\right)^{2}}\right)_{*}: A_{h o m}^{2}\left(S_{b} \times S_{b}\right) \rightarrow A_{h o m}^{2}\left(S_{b} \times S_{b}\right) .
$$

Plugging in the definition of $\Gamma_{0}$, we obtain

$$
\begin{aligned}
\left(\left.\Psi_{b} \circ\left(\Gamma_{0}\right)\right|_{\left(S_{b}\right)^{4}} \circ{ }^{t} \Psi_{b} \circ \Pi_{2}^{\left(S_{b}\right)^{2}}\right)_{*} & =\left(\Psi_{b} \circ\left(\Gamma_{\iota_{b}}+\Delta_{X_{b}}\right) \circ \Pi_{2}^{X_{b}} \circ{ }^{t} \Psi_{b} \circ \Pi_{2}^{\left(S_{b}\right)^{2}}\right)_{*} \\
& =\left(\Psi_{b} \circ\left(\Gamma_{\iota_{b}}+\Delta_{X_{b}}\right) \circ \Pi_{2}^{X_{b}} \circ{ }^{t} \Psi_{b}\right)_{*} \\
& =\left(\left.\Psi_{b} \circ\left(\Gamma_{0}\right)\right|_{X_{b} \times X_{b}} \circ{ }^{t} \Psi_{b}\right)_{*}: \quad A_{h o m}^{2}\left(\left(S_{b}\right)^{2}\right) \rightarrow A_{h o m}^{2}\left(\left(S_{b}\right)^{2}\right),
\end{aligned}
$$

where the second equality is thanks to the relation (12).

This ends the proof of claim 4.6. The next step is the following:

Claim 4.8. For all $b \in B$, we have

$$
\left(\left.\left(\Psi \circ \Gamma_{0} \circ{ }^{t} \Psi+\psi^{\prime}\right)\right|_{\left(S_{b}\right)^{4}}\right)_{*}=0: \quad A_{A J}^{2}\left(S_{b} \times S_{b}\right) \rightarrow A_{A J}^{2}\left(S_{b} \times S_{b}\right),
$$

where $\psi^{\prime}$ is a sum of restrictions of cycles coming from larger varieties as in proposition 4.4.

Proof. This follows from claim 4.6, provided we manage to convince ourselves that

$$
\left.\left.\left(\gamma^{\prime}\right)\right|_{\left(S_{b}\right)^{4}}\right)_{*}=0: \quad A^{4}\left(S_{b} \times S_{b}\right) \rightarrow A^{4}\left(S_{b} \times S_{b}\right) \quad \forall b \in B
$$

For general $b \in B$, (13) is clearly true: by construction, $\gamma^{\prime}$ is supported on $\mathcal{D} \times_{B} \mathcal{D}$, and for general $b$ the fibre $\left(S_{b}\right)^{4}$ will meet the divisor $\mathcal{D}$ in a divisor $D_{b} \subset\left(S_{b}\right)^{2}$; since a 0-cycle on $\left(S_{b}\right)^{2}$ can avoid the divisor $D_{b}$, the restriction $\left.\left(\gamma^{\prime}\right)\right|_{\left(S_{b}\right)^{4}}$ does not act on 0-cycles.

Now let $b_{0} \in B$ be any given point. The Hilbert schemes argument (proposition 4.3) can be made relative to $b_{0}$, to the effect that one obtains a divisor $\mathcal{D}$ in general position with respect to the fibre $\left(S_{b_{0}}\right)^{4}$. As above, one then obtains the vanishing (13) for the fibre over $b_{0}$.

The next step is a further improvement on claim 4.8:

Claim 4.9. For all $b \in B$, we have

$$
\left(\left.\left(\Psi \circ \Gamma_{0} \circ{ }^{t} \Psi\right)\right|_{\left(S_{b}\right)^{4}}\right)_{*}=0: \quad A_{A J}^{2}\left(S_{b} \times S_{b}\right) \rightarrow A_{A J}^{2}\left(S_{b} \times S_{b}\right) .
$$


Proof. This follows from claim 4.8, provided we manage to convince ourselves that

$$
\left(\left.\psi\right|_{\left(S_{b}\right)^{4}}\right)_{*}=0: \quad A_{A J}^{2}\left(S_{b} \times S_{b}\right) \rightarrow A_{A J}^{2}\left(S_{b} \times S_{b}\right) \quad \forall b \in B
$$

where $\psi \in A^{4}\left(\mathcal{S}^{4 / B}\right)$ is a cycle which is coming from larger varieties as in proposition 4.4.

For a given $b \in B$, let us write

$$
\begin{aligned}
& \psi_{b}:=\left.\psi\right|_{\left(S_{b}\right)^{4}} \quad \in A^{4}\left(\left(S_{b}\right)^{4}\right), \\
& \psi_{b, j}:=\left.\left(\psi_{j}\right)\right|_{\left(S_{b}\right)^{4}} \quad \in A^{4}\left(\left(S_{b}\right)^{4}\right) \quad(j=1, \ldots, 6),
\end{aligned}
$$

where

$$
\psi_{b}=\psi_{b, 1}+\cdots+\psi_{b, 6} \text { in } A^{4}\left(\left(S_{b}\right)^{4}\right),
$$

and $\psi_{b, 1}$ (resp. $\psi_{b, 2}, \ldots, \psi_{b, 6}$ ) is the restriction of a cycle on $\mathbb{P}^{3} \times \mathbb{P}^{3} \times S_{b} \times S_{b}$ (resp. on $\mathbb{P}^{3} \times S_{b} \times \mathbb{P}^{3} \times S_{b}, \ldots$, resp. on $\left.S_{b} \times S_{b} \times \mathbb{P}^{3} \times \mathbb{P}^{3}\right)$.

Obviously,

$$
\left(\psi_{b, j}\right)_{*}=0: \quad A_{A J}^{2}\left(S_{b} \times S_{b}\right) \rightarrow A_{A J}^{2}\left(S_{b} \times S_{b}\right) \text { for } j=1,6
$$

(Indeed, the action of $\psi_{b, 1}$ factors over $A_{A J}^{4}\left(\mathbb{P}^{3} \times \mathbb{P}^{3}\right)=0$, and the action of $\psi_{b, 6}$ factors over $\left.A_{A J}^{2}\left(\mathbb{P}^{3} \times \mathbb{P}^{3}\right)=0\right)$.

For the $\psi_{b, j}$ with $j=2, \ldots, 5$, some more work is needed. We will treat the case of $\psi_{b, 2}$ in detail (the argument for the cases $j=3,4,5$ is the same, up to permutation of the factors). Since

$$
A^{4}\left(\mathbb{P}^{3} \times S_{b} \times \mathbb{P}^{3} \times S_{b}\right)=\bigoplus_{k+\ell+m=4} A^{k}\left(S_{b} \times S_{b}\right) \otimes A^{\ell}\left(\mathbb{P}^{3}\right) \otimes A^{m}\left(\mathbb{P}^{3}\right)
$$

we can write $\psi_{b, 2}$ uniquely as a sum

$$
\psi_{b, 2}=\sum_{k+\ell+m=4}\left(h_{b}\right)^{\ell} \times a_{b, k, \ell, m} \times\left(h_{b}\right)^{m} \text { in } A^{4}\left(\left(S_{b}\right)^{4}\right),
$$

where $h_{b} \in A^{1}\left(S_{b}\right)$ is an ample class with $\left(h_{b}\right)^{2}=16$ in $H^{4}\left(S_{b}\right)$, and $a_{b, k, \ell, m} \in A^{k}\left(S_{b} \times S_{b}\right)$ is understood to be in the 2nd and 4th factor. (More precisely, expression (15) should be taken to mean that

$$
\psi_{b, 2}=\sum_{k+\ell+m=4}\left(p_{1}\right)^{*}\left(h_{b}\right)^{\ell} \cdot\left(p_{3}\right)^{*}\left(h_{b}\right)^{m} \cdot\left(p_{24}\right)^{*}\left(a_{b, k, \ell, m}\right) \text { in } A^{4}\left(\left(S_{b}\right)^{4}\right)
$$

where the $p_{i}$ and $p_{24}$ denote the obvious projections.) 
Likewise, the other $\psi_{b, j}$ decompose as sums in $A^{4}\left(\left(S_{b}\right)^{4}\right)$ :

$$
\begin{aligned}
& \psi_{b, 1}=\sum_{k+\ell+m=4}\left(h_{b}\right)^{\ell} \times\left(h_{b}\right)^{m} \times a_{b, k, \ell, m}^{1}, \\
& \psi_{b, 3}=\sum_{k+\ell+m=4}\left(p_{23}\right)^{*}\left(a_{b, k, \ell, m}^{3}\right) \cdot\left(p_{1}\right)^{*}\left(h_{b}\right)^{\ell} \cdot\left(p_{4}\right)^{*}\left(h_{b}\right)^{m}, \\
& \psi_{b, 4}=\sum_{k+\ell+m=4}\left(p_{14}\right)^{*}\left(a_{b, k, \ell, m}^{4}\right) \cdot\left(p_{2}\right)^{*}\left(h_{b}\right)^{\ell} \cdot\left(p_{3}\right)^{*}\left(h_{b}\right)^{m}, \\
& \psi_{b, 5}=\sum_{k+\ell+m=4}\left(p_{13}\right)^{*}\left(a_{b, k, \ell, m}^{5}\right) \cdot\left(p_{2}\right)^{*}\left(h_{b}\right)^{\ell} \cdot\left(p_{4}\right)^{*}\left(h_{b}\right)^{m}, \\
& \psi_{b, 6}=\sum_{k+\ell+m=4} a_{b, k, \ell, m}^{6} \times\left(h_{b}\right)^{\ell} \times\left(h_{b}\right)^{m},
\end{aligned}
$$

where $a_{b, k, \ell, m}^{j} \in A^{k}\left(S_{b} \times S_{b}\right)$.

Lemma 4.10. Let $a_{b, k, \ell, m} \in A^{k}\left(S_{b} \times S_{b}\right)$ be as in expression (15). We have

$$
\left(\psi_{b, 2}\right)_{*}=\left(\left(h_{b}\right)^{2} \times a_{b, 2,2,0} \times\left(h_{b}\right)^{0}\right)_{*}: \quad A_{A J}^{2}\left(S_{b} \times S_{b}\right) \rightarrow A_{A J}^{2}\left(S_{b} \times S_{b}\right) .
$$

Proof. Suppose $(\ell, m) \neq(2,0)$. Thanks to Lieberman's lemma, there is a factorization

$$
\begin{array}{ccc}
A_{A J}^{3}\left(\mathbb{P}^{3} \times S_{b}\right) & \stackrel{\left(h^{\ell} \times a_{b, k, \ell, m} \times h^{m}\right)_{*}}{\longrightarrow} & A_{A J}^{2}\left(\mathbb{P}^{3} \times S_{b}\right) \\
\uparrow & & \downarrow \\
A_{A J}^{2}\left(S_{b} \times S_{b}\right) & \stackrel{\left(\left(h_{b}\right)^{\ell} \times a_{b, k, \ell, m} \times\left(h_{b}\right)^{m}\right)_{*}}{\longrightarrow} & A_{A J}^{2}\left(S_{b} \times S_{b}\right)
\end{array}
$$

(where $h \in A^{1}\left(\mathbb{P}^{3}\right)$ denotes an ample class restricting to $h_{b} \in A^{1}\left(S_{b}\right)$ ). But

$$
A_{A J}^{3}\left(\mathbb{P}^{3} \times S_{b}\right)=A^{1}\left(\mathbb{P}^{3}\right) \otimes A_{A J}^{2}\left(S_{b}\right),
$$

i.e. any $c \in A_{A J}^{3}\left(\mathbb{P}^{3} \times S_{b}\right)$ can be written $c=h \times d$ with $d \in A_{A J}^{2}\left(S_{b}\right)$. It follows that

$$
\left(h^{\ell} \times a_{b, k, \ell, m} \times h^{m}\right)_{*}(c)=\left(h^{\ell} \times h^{m}\right)_{*}(h) \times\left(a_{b, k, \ell, m}\right)_{*}(d)=0 \quad \in A^{2}\left(\mathbb{P}^{3} \times S_{b}\right) \text { for } \ell \neq 2
$$

(since clearly $\left(h^{\ell} \times h^{m}\right)_{*}(h)=0$ in $A^{*}\left(\mathbb{P}^{3}\right)$ for all $\ell \neq 2$ ). Suppose now $\ell=2$, and so (by hypothesis) $m=1$ or 2 . Then

$$
\left(h^{2} \times a_{b, k, 2, m} \times h^{m}\right)_{*}(c) \in A^{m}\left(\mathbb{P}^{3}\right) \otimes A^{2-m}\left(S_{b}\right) \subset A^{2}\left(\mathbb{P}^{3} \times S_{b}\right) .
$$

But $\left(h^{2} \times a_{b, k, 2, m} \times h^{m}\right)_{*}(c)$ is also Abel-Jacobi trivial, and so

$$
\left(h^{2} \times a_{b, k, 2, m} \times h^{m}\right)_{*}(c) \in\left(A^{m}\left(\mathbb{P}^{3}\right) \otimes A^{2-m}\left(S_{b}\right)\right) \cap A_{A J}^{2}\left(\mathbb{P}^{3} \times S_{b}\right)=0 \text { in } A^{2}\left(\mathbb{P}^{3} \times S_{b}\right) .
$$

Lemma 4.11. Let $a_{b, k, \ell, m} \in A^{k}\left(S_{b} \times S_{b}\right)$ be as in expression (15). Then $a_{b, k, \ell, m} \in H^{2 k}\left(S_{b} \times S_{b}\right)$ is in the image of the natural map

$$
\left(A^{k}\left(\mathbb{P}^{3} \times S_{b}\right) \oplus A^{k}\left(S_{b} \times \mathbb{P}^{3}\right)\right) \rightarrow A^{k}\left(S_{b} \times S_{b}\right) \rightarrow H^{2 k}\left(S_{b} \times S_{b}\right),
$$


i.e. there exist $\alpha_{b, k, \ell, m, p} \in A^{p}\left(S_{b}\right)$ and $\alpha_{b, k, \ell, m, p^{\prime}} \in A^{p}\left(S_{b}\right)$ such that there is equality of cycles modulo homological equivalence

$$
a_{b, k, \ell, m}=\sum_{p=0}^{k} \alpha_{b, k, \ell, m, p} \times\left(h_{b}\right)^{k-p}+\sum_{p^{\prime}=0}^{k}\left(h_{b}\right)^{k-p^{\prime}} \times \alpha_{b, k, \ell, m, p^{\prime}} \text { in } H^{2 k}\left(S_{b} \times S_{b}\right) .
$$

Proof. By construction, $\psi_{b} \in A^{4}\left(\left(S_{b}\right)^{4}\right)$ is homologically trivial:

$$
\psi_{b}=\psi_{b, 1}+\psi_{b, 2}+\cdots+\psi_{b, 6}=0 \text { in } H^{8}\left(\left(S_{b}\right)^{4}\right) .
$$

In particular, intersecting and pushing forward we find a vanishing

$$
\frac{1}{16}\left(p_{24}\right)_{*}\left(\psi_{b} \cdot\left(p_{1}\right)^{*}\left(h_{b}\right)^{2-\ell} \cdot\left(p_{3}\right)^{*}\left(h_{b}\right)^{2-m}\right)=0 \text { in } H^{4}\left(S_{b} \times S_{b}\right)
$$

On the other hand,

$$
\begin{aligned}
\frac{1}{16}\left(p_{24}\right)_{*} & \left(\psi_{b} \cdot\left(p_{1}\right)^{*}\left(h_{b}\right)^{2-\ell} \cdot\left(p_{3}\right)^{*}\left(h_{b}\right)^{2-m}\right)=a_{b, k, \ell, m}+ \\
& \frac{1}{16} \sum_{j \neq 2}\left(p_{24}\right)_{*}\left(\psi_{b, j} \cdot\left(p_{1}\right)^{*}\left(h_{b}\right)^{2-\ell} \cdot\left(p_{3}\right)^{*}\left(h_{b}\right)^{2-m}\right) \text { in } A^{2}\left(S_{b} \times S_{b}\right) .
\end{aligned}
$$

Combined with the vanishing (17), we obtain

$$
a_{b, k, \ell, m}=-\frac{1}{16} \sum_{j \neq 2}\left(p_{24}\right)_{*}\left(\psi_{b, j} \cdot\left(p_{1}\right)^{*}\left(h_{b}\right)^{2-\ell} \cdot\left(p_{3}\right)^{*}\left(h_{b}\right)^{2-m}\right) \text { in } H^{4}\left(S_{b} \times S_{b}\right) \text {. }
$$

But we have seen (expressions (16)) that the $\psi_{b, j}$ for $j \neq 2$ contain an element $\left(h_{b}\right)^{i}$ in either the 2 nd or 4 th factor, and so this proves lemma 4.11. More in detail: let us consider $j=1$. Using 
(16), we find

$$
\begin{aligned}
\left(p_{24}\right)_{*} & \left(\psi_{b, 1} \cdot\left(p_{1}\right)^{*}\left(h_{b}\right)^{2-\ell} \cdot\left(p_{3}\right)^{*}\left(h_{b}\right)^{2-m}\right) \\
& =\left(p_{24}\right)_{*}\left(\sum_{k+\ell^{\prime}+m^{\prime}=4}\left(\left(h_{b}\right)^{\ell^{\prime}} \times\left(h_{b}\right)^{m^{\prime}} \times a_{b, k, \ell^{\prime}, m^{\prime}}^{1}\right) \cdot\left(p_{1}\right)^{*}\left(h_{b}\right)^{2-\ell} \cdot\left(p_{3}\right)^{*}\left(h_{b}\right)^{2-m}\right) \\
& =\sum_{k+\ell^{\prime}+m^{\prime}=4}\left(p_{24}\right)_{*}\left(\left(\left(h_{b}\right)^{2-\ell+\ell^{\prime}} \times\left(h_{b}\right)^{m^{\prime}} \times a_{b, k, \ell^{\prime}, m^{\prime}}^{1}\right) \cdot\left(p_{3}\right)^{*}\left(h_{b}\right)^{2-m}\right) \\
& =\sum_{k+\ell^{\prime}+m^{\prime}=4}\left(p_{24}\right)_{*}\left(\left(\left(h_{b}\right)^{2} \times\left[S_{b}\right] \times a_{b, k, \ell, m^{\prime}}^{1}\right) \cdot\left(p_{3}\right)^{*}\left(h_{b}\right)^{2-m} \cdot\left(p_{24}\right)^{*}\left(\left(h_{b}\right)^{m^{\prime}} \times\left[S_{b}\right]\right)\right) \\
& \left.=\sum_{k+\ell+m^{\prime}=4}\left(p_{24}\right)_{*}\left(\left(h_{b}\right)^{2} \times\left[S_{b}\right] \times a_{b, k, \ell, m^{\prime}}^{1}\right) \cdot\left(p_{3}\right)^{*}\left(h_{b}\right)^{2-m}\right) \cdot\left(\left(h_{b}\right)^{m^{\prime}} \times\left[S_{b}\right]\right) \\
& =\sum_{k+\ell+m^{\prime}=4}\left(p_{24}\right)_{*}\left(\left(h_{b}\right)^{2} \times\left[S_{b}\right] \times(\text { something })\right) \cdot\left(\left(h_{b}\right)^{m^{\prime}} \times\left[S_{b}\right]\right) \\
& =\sum_{k+\ell+m^{\prime}=4}\left(\left[S_{b}\right] \times(\text { something })\right) \cdot\left(\left(h_{b}\right)^{m^{\prime}} \times\left[S_{b}\right]\right) \\
= & \sum_{k+\ell+m^{\prime}=4}\left(h_{b}\right)^{m^{\prime}} \times(\text { something }) \text { in } A^{2}\left(S_{b} \times S_{b}\right) .
\end{aligned}
$$

This shows that

$$
\left(p_{24}\right)_{*}\left(\psi_{b, 1} \cdot\left(p_{1}\right)^{*}\left(h_{b}\right)^{2-\ell} \cdot\left(p_{3}\right)^{*}\left(h_{b}\right)^{2-m}\right)
$$

can be written in the form of the right-hand-side of lemma 4.11. The proof for the other $\psi_{b, j}$ is similar.

We now upgrade (a weak version of the $k=2$ part of) the equality of lemma 4.11 to rational equivalence:

Lemma 4.12. Let $a_{b, k, \ell, m} \in A^{k}\left(S_{b} \times S_{b}\right)$ be as in expression (15). Then $a_{b, 2, \ell, m} \in A^{2}\left(S_{b} \times S_{b}\right)$ can be written

$$
a_{b, 2, \ell, m}=\gamma_{b, 2, \ell, m, 0}+\gamma_{b, 2, \ell, m, 1}+\gamma_{b, 2, \ell, m, 2} \text { in } A^{2}\left(S_{b} \times S_{b}\right),
$$

where $\gamma_{b, 2, \ell, m, j}$ is supported on $V_{b, 2, \ell, m, j} \times W_{b, 2, \ell, m, j}$ for $j=0,1,2$, and $V_{b, 2, \ell, m, j} \subset S_{b}$ is closed of codimension $j$ and $W_{b, 2, \ell, m, j} \subset S_{b}$ is closed of codimension $2-j$.

Proof. This is another application of the technique of "spread" developed in [42], [43]. The application in this instance is easier than the above, for we only need to reason on the fibre product $\mathcal{S} \times{ }_{B} \mathcal{S}$, and $n o t$ on the fourfold relative fibre product $\mathcal{S}^{4 / B}$.

The first thing to do is to find a relative cycle inducing the $a_{b, 2, \ell, m}$ for the various $b$. This can be done as follows: let us define

$$
a_{2, \ell, m}:=\frac{1}{16}\left(p_{24}\right)_{*}\left(\psi_{2} \cdot\left(p_{1}\right)^{*}\left(H^{2-\ell}\right) \cdot\left(p_{3}\right)^{*}\left(H^{2-m}\right)\right) \in A^{2}\left(\mathcal{S} \times_{B} \mathcal{S}\right) .
$$


(Here $H \in A^{1}(\mathcal{S})$ denotes a relatively ample class with $\left.\left(H^{2}\right)\right|_{S_{b}}=16$ in $H^{4}\left(S_{b}\right)$, and $p_{i}, p_{24}$ denote the obvious projections.) The relative cycle $a_{2, \ell, m}$ has the property that the restriction to a fibre is

$\left.a_{2, \ell, m}\right|_{\left(S_{b}\right)^{2}}=\frac{1}{16}\left(p_{24}\right)_{*}\left(\psi_{b, 2} \cdot\left(p_{1}\right)^{*}\left(\left(h_{b}\right)^{2-\ell}\right) \cdot\left(p_{3}\right)^{*}\left(\left(h_{b}\right)^{2-m}\right)\right)=a_{b, 2, \ell, m} \in A^{2}\left(S_{b} \times S_{b}\right) \quad \forall b \in B$, in view of expression (15).

The next thing to do is to find a fibrewise homological property of the relative cycle. Lemma 4.11 implies (after regrouping of the summands) that for each $b \in B$, there exist closed subvarieties $V_{b, 2, \ell, m, j}$ and $W_{b, 2, \ell, m, j} \subset S_{b}$ of codimension $j$ resp. $2-j(j=0,1,2)$, and cycles

$$
\gamma_{b, 2, \ell, m, j} \in A_{2}\left(V_{b, 2, \ell, m, j} \times W_{b, 2, \ell, m, j}\right),
$$

such that

$$
\left.a_{2, \ell, m}\right|_{\left(S_{b}\right)^{2}}=\gamma_{b, 2, \ell, m, 0}+\gamma_{b, 2, \ell, m, 1}+\gamma_{b, 2, \ell, m, 2} \text { in } H^{4}\left(S_{b} \times S_{b}\right) .
$$

Applying proposition 4.13 below, these fibrewise cycles can be spread out to the family: there exist subvarieties

$$
\mathcal{V}_{2, \ell, m, j} \subset \mathcal{S}, \quad \mathcal{W}_{2, \ell, m, j} \subset \mathcal{S}
$$

of codimension $j$ resp. $2-j$, and relative cycles

$$
\gamma_{2, \ell, m, j} \in A_{*}\left(\mathcal{V}_{2, \ell, m, j} \times_{B} \mathcal{W}_{2, \ell, m, j}\right) \quad(j=0,1,2),
$$

such that for each $b \in B$, there is a homological equivalence

$$
\left.a_{2, \ell, m}\right|_{\left(S_{b}\right)^{2}}=\left.\left(\gamma_{2, \ell, m, 0}+\gamma_{2, \ell, m, 1}+\gamma_{2, \ell, m, 2}\right)\right|_{\left(S_{b}\right)^{2}} \text { in } H^{4}\left(S_{b} \times S_{b}\right) .
$$

In other words, the relative cycle

$$
C_{0}:=a_{2, \ell, m}-\gamma_{2, \ell, m, 0}+\gamma_{2, \ell, m, 1}+\gamma_{2, \ell, m, 2} \in A^{2}\left(\mathcal{S} \times{ }_{B} \mathcal{S}\right)
$$

has the property of being homologically trivial on every fibre:

$$
\left.C_{0}\right|_{S_{b} \times S_{b}}=0 \text { in } H^{4}\left(S_{b} \times S_{b}\right), \quad \forall b \in B .
$$

Applying the Leray spectral sequence argument [42, Lemmas 3.11 and 3.12], one can render $C_{0}$ globally homologically trivial, i.e.

$$
C_{1}:=C_{0}+\theta=0 \text { in } H^{4}\left(\mathcal{S} \times{ }_{B} \mathcal{S}\right),
$$

where $\theta \in A^{2}\left(\mathcal{S} \times{ }_{B} \mathcal{S}\right)$ is the restriction of a cycle in $A^{2}\left(B \times \mathbb{P}^{3} \times \mathbb{P}^{3}\right)$. But

$$
A_{\text {hom }}^{2}\left(\mathcal{S} \times_{B} \mathcal{S}\right)=0
$$

([42, Proposition 3.13], combined with the fact that the Voisin standard conjecture [42, Conjecture 1.6] is true in codimension 2), and so $C_{1}$ is rationally trivial. In particular, restricting to a fibre one obtains

$$
\left.\left(C_{0}+\theta\right)\right|_{S_{b} \times S_{b}}=0 \quad \text { in } A^{2}\left(S_{b} \times S_{b}\right), \quad \forall b \in B .
$$

The restriction $\left.\theta\right|_{S_{b} \times S_{b}}$ (coming from $A^{2}\left(\mathbb{P}^{3} \times \mathbb{P}^{3}\right)$ ) is of the form $\sum_{j}\left(h_{b}\right)^{j} \times\left(h_{b}\right)^{2-j}$. Thus (after modifying the $V_{b, 2, \ell, m, j}$ and $W_{b, 2, \ell, m, j}$, we find that

$$
\left.C_{0}\right|_{S_{b} \times S_{b}}=\left.\left(a_{2, \ell, m}-\gamma_{2, \ell, m, 0}-\gamma_{2, \ell, m, 1}-\gamma_{2, \ell, m, 2}\right)\right|_{S_{b} \times S_{b}}=0 \quad \text { in } A^{2}\left(S_{b} \times S_{b}\right), \quad \forall b \in B,
$$


proving lemma 4.12 .

(Alternatively, using the approach of [43], one could forsake the Leray spectral sequence argument, and skip directly from equality (18) to equality (19) by invoking [43, Proposition 1.6].)

Proposition 4.13. Let $\mathcal{X} \rightarrow B$ be a smooth projective morphism of relative dimension $n$, and let $\Gamma \in A^{n}\left(\mathcal{X} \times_{B} \mathcal{X}\right)$. Assume that for the very general $b \in B$, there exist closed subvarieties $V_{b, j} \subset X_{b}, W_{b, j} \subset X_{b}$ of codimension $j$ resp. $n-j$, and cycles $\gamma_{b, j} \in A_{n}\left(V_{b, j} \times W_{b, j}\right)$ such that

$$
\left.\Gamma\right|_{X_{b} \times X_{b}}=\gamma_{b, 0}+\cdots+\gamma_{b, n} \text { in } H^{2 n}\left(X_{b} \times X_{b}\right) \text {. }
$$

Then there exist closed subvarieties $\mathcal{V}_{j} \subset \mathcal{X}, \mathcal{W}_{j} \subset \mathcal{X}$ of codimension $j$ resp. $n-j$, and cycles $\gamma_{j} \in A_{*}\left(\mathcal{V}_{j} \times{ }_{B} \mathcal{W}_{j}\right)$, such that

$$
\left.\Gamma\right|_{X_{b} \times X_{b}}=\left.\left(\gamma_{0}+\cdots+\gamma_{n}\right)\right|_{X_{b} \times X_{b}} \text { in } H^{2 n}\left(X_{b} \times X_{b}\right),
$$

for all $b \in B$.

Proof. This is the same Hilbert schemes argument as [42, Proposition 3.7] (i.e., proposition 4.3 above). The point is that the data of all the $V_{b, j}, W_{b, j}, \gamma_{b, j}$ can be encoded by a countably infinite set of varieties. Since by assumption, this countably infinite set dominates $B$, one of the varieties must dominate $B$.

We are now in position to wrap up the proof of claim 4.9. We have

$$
\begin{aligned}
\left(\psi_{b, 2}\right)_{*} & =\left(\left(h_{b}\right)^{2} \times a_{b, 2,2,0} \times\left[S_{b}\right]\right)_{*} \\
& =\left(\left(h_{b}\right)^{2} \times\left(\sum_{j=0}^{2} \gamma_{b, 2,2,0, j}\right) \times\left[S_{b}\right]\right) * \\
& \stackrel{(*)}{=} 0: A_{A J}^{2}\left(\left(S_{b}\right)^{2}\right) \rightarrow A_{A J}^{2}\left(\left(S_{b}\right)^{2}\right) .
\end{aligned}
$$

Here, the first equality is lemma 4.10, and the second equality is lemma 4.12. As for the equality labelled $(*)$, this is true for dimension reasons: indeed, there is a factorization

$$
\begin{array}{ccc}
A_{A J}^{2}\left(\left(h_{b}\right)^{2} \times \widehat{V_{b, 2, \ell, m, j}}\right) & \rightarrow & A_{A J}^{j}\left(\widehat{W_{b, 2, \ell, m, j}} \times S_{b}\right) \\
\uparrow & & \downarrow \\
A_{A J}^{2}\left(S_{b} \times S_{b}\right) & \stackrel{\left(\left(h_{b}\right)^{2} \times \gamma_{b, 2,2,0, j} \times\left[S_{b}\right]\right)_{*}}{\longrightarrow} & A_{A J}^{2}\left(S_{b} \times S_{b}\right) .
\end{array}
$$

(Here the $\widetilde{V}$ and $\widetilde{W}$ denote resolutions of singularities.) The upper-right corner $A_{A J}^{j}()$ is 0 unless $j=2$. However, for $j=2$ the dimension of $\left(h_{b}\right)^{2} \times \widetilde{V_{b, 2, \ell, m, j}}$ is 0 and so in this case the upper-left corner is 0 . This proves equality $(*)$ for general $b \in B$. For any given $b_{0} \in B$, the cycles $\gamma_{2,2,0, j}$ can be moved in general position with respect to the fibre $S_{b_{0}} \times S_{b_{0}}$, and then the above argument applies to prove $(*)$ for $S_{b_{0}}$.

We have now proven that the correspondence $\psi_{b, 2}$ does not act on $A_{A J}^{2}\left(\left(S_{b}\right)^{2}\right)$ for all $b \in$ $B$. The same argument also proves that the correspondences $\psi_{b, j}, j=3,4,5$ do not act on $A_{A J}^{2}\left(\left(S_{b}\right)^{2}\right)$ (the argument is only notationally different), and so

$$
\left(\psi_{b}\right)_{*}=0: \quad A_{A J}^{2}\left(\left(S_{b}\right)^{2}\right) \rightarrow A_{A J}^{2}\left(\left(S_{b}\right)^{2}\right) \quad \forall b \in B .
$$


This proves equality (14), and hence also claim 4.9 .

The last step is to return from the product $S_{b} \times S_{b}$ to the Hilbert scheme $X_{b}$ :

Claim 4.14. For all $b \in B$, we have

$$
\left.\left(\left.\left(\Gamma_{0}\right)\right|_{X_{b} \times X_{b}}\right)\right)_{*}=0: \quad A_{A J}^{2}\left(X_{b}\right) \rightarrow A^{2}\left(X_{b}\right) .
$$

Proof. This is immediate from claim 4.9, since

$$
\left({ }^{t} \Psi_{b}\right)_{*}\left(\Psi_{b}\right)_{*}=\mathrm{id}: A_{A J}^{2}\left(X_{b}\right) \rightarrow A_{A J}^{2}\left(X_{b}\right) .
$$

By definition of $\Gamma_{0}$, claim 4.14 implies that

$$
\left(\Gamma_{\iota_{b}}+\Delta_{X_{b}}\right)_{*}\left(\Pi_{2}^{X_{b}}\right)_{*}=0: \quad A_{A J}^{2}\left(X_{b}\right) \rightarrow A^{2}\left(X_{b}\right) \quad \forall b \in B .
$$

But $\Pi_{2}^{X_{b}}$ is a projector on $A_{(2)}^{2}\left(X_{b}\right) \subset A_{A J}^{2}\left(X_{b}\right)$ and so

$$
\left(\Gamma_{\iota b}+\Delta_{X_{b}}\right)_{*}=0: \quad A_{(2)}^{2}\left(X_{b}\right) \rightarrow A^{2}\left(X_{b}\right) \quad \forall b \in B,
$$

which concludes the proof of theorem 4.2.

4.2. Action on $A^{4}$. In this subsection, we finish the proof of our main result (theorem 4.1), by checking that the involution $\iota$ has the expected action on $A^{4}$ :

Theorem 4.15. Let $X$ and $\iota$ be as in theorem 4.1. Then

$$
\iota^{*}: \quad A_{(j)}^{4}(X) \rightarrow A^{4}(X)= \begin{cases}\mathrm{id} & \text { if } j=0,4 ; \\ -\mathrm{id} & \text { if } j=2 .\end{cases}
$$

Proof. The case $j=0$ is easy: there is a $\iota$-invariant ample divisor $D$ (proposition 2.21). As $D$ is ample, the intersection $D^{4}$ is non-zero and so (since $D^{4} \in A_{(0)}^{4}$, and $A_{(0)}^{4}(X)$ is onedimensional)

$$
A_{(0)}^{4}(X)=\mathbb{Q}\left[D^{4}\right]
$$

We now consider the case $j=4$. As we have seen (theorem 2.17), Shen-Vial have proven the multiplication map

$$
A_{(2)}^{2}(X) \otimes A_{(2)}^{2}(X) \rightarrow A_{(4)}^{4}(X)
$$

is surjective. Given $b \in A_{(4)}^{4}(X)$, we can thus write

$$
b=a_{1} \cdot a_{2} \text { in } A^{4}(X),
$$

where $a_{1}, a_{2} \in A_{(2)}^{2}(X)$. But then, using theorem 4.2 we find

$$
\iota^{*}(b)=\iota^{*}\left(a_{1}\right) \cdot \iota^{*}\left(a_{2}\right)=\left(-a_{1}\right) \cdot\left(-a_{2}\right)=a_{1} \cdot a_{2}=b \text { in } A^{4}(X) .
$$

It remains to prove theorem 4.15 for $j=2$. As we have seen (theorem 2.17), Shen-Vial have established an isomorphism

$$
\cdot l: \quad A_{(2)}^{2}(X) \stackrel{\cong}{\rightarrow} A_{(2)}^{4}(X) .
$$


Theorem 4.15 now follows, provided we understand the action of $\iota$ on the class $l \in A^{2}(X)$. To this end, we will prove the following:

Proposition 4.16. Let $X$ and $\iota$ be as in theorem 4.1. Let $l \in A_{(0)}^{2}(X)$ be the class as in theorem 2.17. Then

$$
\iota^{*}(l)= \pm l \text { in } A^{2}(X) .
$$

Proposition 4.16 suffices to prove theorem 4.1. Indeed, let us suppose for a moment that

$$
\iota^{*}(l)=-l \text { in } A^{2}(X) .
$$

Using the isomorphism (20) and theorem 4.2, this would imply

$$
\iota^{*}=\mathrm{id}: \quad A_{(2)}^{4}(X) \rightarrow A^{4}(X) .
$$

Since $\iota$ acts as the identity on $A_{(j)}^{4}(X)$ for $j=0,4$, this would imply

$$
\iota^{*}=\mathrm{id}: \quad A^{4}(X) \rightarrow A^{4}(X) .
$$

Using the Bloch-Srinivas argument [7] applied to $\Gamma_{\iota}-\Delta_{X}$, this would imply that

$$
\Gamma_{\iota}-\Delta_{X}=\gamma \text { in } A^{4}(X \times X),
$$

where $\gamma$ is a cycle supported on $X \times D$ for $D \subset X$ a divisor. In particular, this would imply

$$
\iota^{*}=\mathrm{id}: \quad H^{2,0}(X) \rightarrow H^{2,0}(X),
$$

which is absurd since we know that $\iota$ is non-symplectic. The minus sign in proposition 4.16 can thus be excluded; assuming proposition 4.16 is true, we must have $\iota^{*}(l)=l$.

Now let $c \in A_{(2)}^{4}(X)$. Using the isomorphism (20), we can find $a \in A_{(2)}^{2}(X)$ such that

$$
c=l \cdot a \text { in } A^{4}(X) \text {. }
$$

But then

$$
\iota^{*}(c)=\iota^{*}(l) \cdot \iota^{*}(a)=l \cdot(-a)=-l \cdot a=-c \text { in } A^{4}(X) .
$$

Here, the second equality comes from proposition 4.16 and theorem 4.2. This proves theorem 4.1, assuming proposition 4.16 .

We now proceed with the proof of proposition 4.16. The first step is to prove the statement in homology:

Lemma 4.17. Let $S$ be any $K 3$ surface and let $X=S^{[2]}$. Let $l \in A^{2}(X)$ be the class of theorem 2.17, and let $\iota \in \operatorname{Aut}(X)$ be an involution. We have

$$
\iota^{*}(l)= \pm l \text { in } H^{4}(X) .
$$

Proof. Shen and Vial have constructed a distinguished cycle $L \in A^{2}(X \times X)$ (whose cohomology class is the Beauville-Bogomolov class denoted $\mathfrak{B}$ in loc. cit.), and an eigenspace decomposition

$$
A^{2}(X)=\Lambda_{25}^{2} \oplus \Lambda_{2}^{2} \oplus \Lambda_{0}^{2}
$$

where

$$
\Lambda_{\lambda}^{i}:=\left\{a \in A^{i}(X) \mid\left(L^{2}\right)_{*}(a)=\lambda a\right\}
$$


and

$$
\Lambda_{25}^{2}=\mathbb{Q}[l]
$$

(This is [35, Theorem 14.5, Propositions 14.6 and 14.8], combined with [35, Theorem 2.2]).

We now observe the following commutativity relation in cohomology:

Lemma 4.18. Set-up as in lemma 4.17. Then

$$
\left(L^{2}\right)_{*} \iota^{*}=\iota^{*}\left(L^{2}\right)_{*}: \quad H^{i}(X) \rightarrow H^{i}(X) .
$$

Proof. Let $L \in A^{2}(X \times X)$ be the Shen-Vial cycle as above. As proven in [35, Proposition 1.3(i)], the Shen-Vial cycle satisfies a quadratic relation

$$
L^{2}=2 \Delta_{X}-\frac{2}{25}\left(l_{1}+l_{2}\right) L-\frac{1}{23 \cdot 25}\left(2 l_{1}^{2}-23 l_{1} l_{2}+2 l_{2}^{2}\right) \text { in } H^{8}(X \times X),
$$

where $l:=\left(i_{\Delta}\right)^{*}(L)$ (and $i_{\Delta}: X \rightarrow X \times X$ is the diagonal embedding) and $l_{i}:=\left(p_{i}\right)^{*}(l)$ (and $p_{i}$ are the obvious projections).

Let us define a modified cycle

$$
L^{\prime}:=\Gamma_{\iota} \circ L \circ \Gamma_{\iota} \in A^{2}(X \times X) .
$$

Using Lieberman's lemma [37, Lemma 3.3] plus the fact that ${ }^{t} \Gamma_{\iota}=\Gamma_{\iota}$, we see that

$$
L^{\prime}=(\iota \times \iota)^{*}(L) \text { in } A^{2}(X \times X) \text {. }
$$

Define also $l^{\prime}:=\left(i_{\Delta}\right)^{*}\left(L^{\prime}\right) \in A^{2}(X)$ and $l_{i}^{\prime}:=\left(p_{i}\right)^{*}\left(l^{\prime} \in A^{2}(X \times X), i=1,2\right.$. Since the diagram

commutes, we have the relations

$$
\begin{aligned}
& X \times X \quad \stackrel{p_{i}}{\rightarrow} \quad X \quad \stackrel{i_{\Delta}}{\rightarrow} \quad X \times X \\
& \downarrow \iota \times \iota \quad \downarrow \iota \quad \downarrow \iota \times \iota \\
& X \times X \quad \stackrel{p_{i}}{\rightarrow} \quad X \quad \stackrel{i_{\Delta}}{\rightarrow} \quad X \times X
\end{aligned}
$$

$$
l_{i}^{\prime}=(\iota \times \iota)^{*}\left(l_{i}\right) \text { in } A^{2}(X \times X), \quad i=1,2 .
$$

Let us apply $(\iota \times \iota)^{*}$ to the quadratic relation (22). The result is a relation

$$
\begin{array}{r}
(\iota \times \iota)^{*}\left(L^{2}\right)=2 \Delta_{X}-\frac{2}{25}(\iota \times \iota)^{*}\left(l_{1}+l_{2}\right) L^{\prime}-\frac{1}{23 \cdot 25}(\iota \times \iota)^{*}\left(2 l_{1}^{2}-23 l_{1} l_{2}+2 l_{2}^{2}\right) \\
\text { in } H^{8}(X \times X) .
\end{array}
$$

But

$$
(\iota \times \iota)^{*}\left(L^{2}\right)=\left((\iota \times \iota)^{*} L\right)^{2}=\left(L^{\prime}\right)^{2} \text { in } A^{4}(X \times X) .
$$

Plugging this in equality (24), and also using the relations (23), we find that the cycle $L^{\prime}$ satisfies a quadratic relation

$$
\left(L^{\prime}\right)^{2}=2 \Delta_{X}-\frac{2}{25}\left(l_{1}^{\prime}+l_{2}^{\prime}\right) L^{\prime}-\frac{1}{23 \cdot 25}\left(2\left(l_{1}^{\prime}\right)^{2}-23 l_{1}^{\prime} l_{2}^{\prime}+2\left(l_{2}^{\prime}\right)^{2}\right) \text { in } H^{8}(X \times X) .
$$

But then, applying the unicity result [35, Proposition $1.3(\mathrm{v})]$, we find there is equality

$$
L^{\prime}= \pm L \text { in } H^{4}(X \times X) \text {. }
$$


In particular, there is equality

$$
\left(L^{\prime}\right)^{2}=L^{2} \text { in } H^{8}(X \times X) .
$$

In view of equality (25), this means

$$
\Gamma_{\iota} \circ\left(L^{2}\right) \circ \Gamma_{\iota}=L^{2} \text { in } H^{8}(X \times X),
$$

and so (by composing with $\Gamma_{\iota}$ )

$$
\Gamma_{\iota} \circ\left(L^{2}\right)=\left(L^{2}\right) \circ \Gamma_{\iota} \text { in } H^{4}(X \times X) .
$$

This proves lemma 4.18 .

The eigenspace decomposition (21) induces an eigenspace decomposition modulo homological equivalence:

$$
\operatorname{Im}\left(A^{2}(X) \rightarrow H^{4}(X)\right)=\Lambda_{25}^{2}+\frac{\Lambda_{2}^{2}}{A_{(0)}^{2}(X) \cap A_{h o m}^{2}(X)}
$$

(this is the algebraic part of the eigenspace decomposition of $H^{4}(X)$ given in [35, Proposition 1.3(iii)]).

Lemma (4.18) implies $\iota$ preserves this eigenspace decomposition modulo homological equivalence. In particular, $\iota^{*} \Lambda_{25}^{2} \subset \Lambda_{25}^{2}$ (modulo homologically trivial cycles), and so

$$
\iota^{*}(l)=d l \text { in } H^{4}(X),
$$

for some $d \in \mathbb{Q}$. Since $A_{(0)}^{4}(X)=\mathbb{Q}\left[l^{2}\right][35$, Theorem 4.6], we have

$$
\iota^{*}\left(l^{2}\right)=l^{2} \text { in } H^{8}(X),
$$

and so $d= \pm 1$. This proves lemma 4.17 .

The next step (in proving proposition 4.16) is to upgrade to rational equivalence. Here, we use again the method of "spread" developed in [42], [43]. As in the proof of theorem 4.2, let $\mathcal{S} \rightarrow B$ resp. $\mathcal{X} \rightarrow B$ denote the family of all smooth quartics $S_{b} \subset \mathbb{P}^{3}$ with Picard number 1 , resp. of all Hilbert schemes $X_{b}=\left(S_{b}\right)^{[2]}$. We note that there exists a relative cycle

$$
\mathcal{L} \in A^{2}(\mathcal{X})
$$

such that restriction

$$
\left.\mathcal{L}\right|_{X_{b}}=l_{b} \quad \in A^{2}\left(X_{b}\right) \quad \forall b \in B
$$

is the distinguished class (denoted $l$ in theorem 2.17) for the fibre $X_{b}$. Indeed, one defines $\mathcal{L}$ as

$$
\mathcal{L}:=\frac{5}{6} c_{2}\left(T_{\mathcal{X} / B}\right) \quad \in A^{2}(\mathcal{X})
$$

where $T_{\mathcal{X} / B}$ is the relative tangent bundle of the smooth morphism $\mathcal{X} \rightarrow B$. Since for any $b \in B$ there is a relation

[35, Equation (93)], this implies (27).

$$
l_{b}=\frac{5}{6} c_{2}\left(X_{b}\right) \text { in } A^{2}\left(X_{b}\right)
$$


The relative cycle

$$
\Gamma_{0}:=\mathcal{L} \pm \Gamma_{\iota} \circ \mathcal{L} \quad \in A^{2}(\mathcal{X})
$$

is such that the restriction to each fibre is homologically trivial:

$$
\left.\left(\Gamma_{0}\right)\right|_{X_{b}}=0 \text { in } H^{4}\left(X_{b}\right) \text {. }
$$

(Here, " \pm " is taken to mean + (resp. - ) if lemma 4.17 is true with a + (resp. a - ).) Thus, the relative cycle

$$
\Gamma_{1}:=\Psi_{*}\left(\Gamma_{0}\right) \quad \in A^{2}\left(\mathcal{S} \times{ }_{B} \mathcal{S}\right)
$$

also is homologically trivial on each fibre. (Here, $\Psi$ is the relative correspondence from $\mathcal{X}$ to $\mathcal{S} \times{ }_{B} \mathcal{S}$ as in the proof of theorem 4.2.)

Applying [42, Lemma 3.12], up to shrinking $B$ we can make $\Gamma_{1}$ globally homologically trivial. That is, there exists

$$
\psi \in \operatorname{Im}\left(A^{2}\left(B \times \mathbb{P}^{3} \times \mathbb{P}^{3}\right) \rightarrow A^{2}\left(\mathcal{S} \times{ }_{B} \mathcal{S}\right)\right)
$$

such that (after replacing $B$ by a non-empty open subset $B^{\prime} \subset B$ )

$$
\Gamma_{2}:=\Gamma_{1}+\psi \quad \in A^{2}\left(\mathcal{S} \times{ }_{B^{\prime}} \mathcal{S}\right)
$$

is actually in $A_{\text {hom }}^{2}\left(\mathcal{S} \times{ }_{B^{\prime}} \mathcal{S}\right)$.

But $A_{\text {hom }}^{2}\left(\mathcal{S} \times_{B^{\prime}} \mathcal{S}\right)=0$ (this follows from [42, Proposition 3.13], combined with the fact that the "Voisin standard conjecture" [42, Conjecture 1.6] is known to hold in codimension 2), and so

$$
\Gamma_{2}=0 \quad \in A^{2}\left(\mathcal{S} \times B^{\prime} \mathcal{S}\right) .
$$

Restricting to a fibre, we find

$$
\left.\left(\Gamma_{1}\right)\right|_{S_{b} \times S_{b}}+\left.\psi\right|_{S_{b} \times S_{b}}=0 \text { in } A^{2}\left(S_{b} \times S_{b}\right) \quad \forall b \in B^{\prime} .
$$

As $\Gamma_{1}$ is fibrewise homologically trivial, the same goes for $\psi$ :

$$
\left.\psi\right|_{S_{b} \times S_{b}}=0 \text { in } H^{4}\left(S_{b} \times S_{b}\right) \quad \forall b \in B^{\prime} .
$$

But $A^{2}\left(\mathbb{P}^{3} \times \mathbb{P}^{3}\right)=\oplus_{i} A^{i}\left(\mathbb{P}^{3}\right) \otimes A^{2-i}\left(\mathbb{P}^{3}\right)$ and so

$$
\left.\psi\right|_{S_{b} \times S_{b}}=\lambda_{0}\left[S_{b}\right] \times H_{b}^{2}+\lambda_{1} H_{b} \times H_{b}+\lambda_{2} H_{b}^{2} \times\left[S_{b}\right] \text { in } A^{2}\left(S_{b} \times S_{b}\right),
$$

where $\lambda_{i} \in \mathbb{Q}$ and $H_{b} \in A^{1}\left(S_{b}\right)$ is an ample class on $S_{b}$. It follows from the vanishing (28) that the $\lambda_{i}$ must be 0 , and so $\left.\psi\right|_{S_{b} \times S_{b}}$ is rationally trivial, and hence also

$$
\left.\left(\Gamma_{1}\right)\right|_{S_{b} \times S_{b}}=0 \text { in } A^{2}\left(S_{b} \times S_{b}\right) .
$$

Composing with ${ }^{t} \Psi_{b}$, it follows that also

$$
\left({ }^{t} \Psi_{b}\right)_{*}\left(\left.\left(\Gamma_{1}\right)\right|_{S_{b} \times S_{b}}\right)=\left({ }^{t} \Psi_{b}\right)_{*}\left(\Psi_{b}\right)_{*}\left(\left.\left(\Gamma_{0}\right)\right|_{X_{b}}\right)=0 \text { in } A^{2}\left(X_{b}\right) \quad \forall b \in B^{\prime} .
$$

On the other hand, as we have seen above $\left.\left(\Gamma_{0}\right)\right|_{X_{b}} \in A_{h o m}^{2}\left(X_{b}\right)$ and $\left({ }^{t} \Psi_{b}\right)_{*}\left(\Psi_{b}\right)_{*}$ is the identity on $A_{h o m}^{2}\left(X_{b}\right)$. It follows that

$$
\left.\left(\Gamma_{0}\right)\right|_{X_{b}}=\left.\left(l_{b} \pm\left(\iota_{b}\right)^{*}\left(l_{b}\right)\right)\right|_{X_{b}}=0 \text { in } A^{2}\left(X_{b}\right) \quad \forall b \in B^{\prime} .
$$

This proves proposition 4.16 for general $b \in B$. To extend to all $b \in B$, one can invoke [45, Lemma 3.2]. Proposition 4.16 and theorem 4.15 are now proven.

For later use, we remark that the above argument also proves the following statement: 
Corollary 4.19. Let $X$ and $\iota$ be as in theorem 4.1. Then

$$
\iota^{*} A_{(0)}^{2}(X) \subset A_{(0)}^{2}(X) .
$$

Proof. Let $b \in A_{(0)}^{2}(X)$, and suppose

$$
\iota^{*}(b)=c_{0}+c_{2} \text { in } A^{2}(X),
$$

with $c_{0} \in A_{(0)}^{2}(X)$ and $c_{2} \in A_{(2)}^{2}(X)$.

Let $l \in A_{(0)}^{2}(X)$ be the distinguished class of theorem 2.17. The 0 -cycle $b \cdot l$ is in $A_{(0)}^{4}(X)$, and so

$$
\iota^{*}(b \cdot l)=b \cdot l \text { in } A_{(0)}^{4}(X)
$$

On the other hand, we have

$$
\iota^{*}(b \cdot l)=\iota^{*}(b) \cdot \iota^{*}(l)=\left(c_{0}+c_{2}\right) \cdot l=c_{0} \cdot l+c_{2} \cdot l \text { in } A^{4}(X) .
$$

(Here we have used proposition 4.16, which we have seen must be true with a + sign.) Since $c_{0} \cdot l \in A_{(0)}^{4}(X)$ and $c_{2} \cdot l \in A_{(2)}^{4}(X)$, we must have

$$
c_{0} \cdot l=b \cdot l \quad \text { in } A_{(0)}^{4}(X), \quad c_{2} \cdot l=0 \text { in } A_{(2)}^{4}(X) .
$$

Using the injectivity part of theorem 2.17, this implies that $c_{2}=0$.

Remark 4.20. Another way of proving the $j=2$ case of theorem 4.15 could be as follows: define a relative correspondence

$$
\Gamma_{0}^{\prime}:=\Pi_{6}^{\mathcal{X}} \circ\left(\Gamma_{\iota}+\Delta_{\mathcal{X}}\right) \quad \in A^{4}\left(\mathcal{X} \times_{B} \mathcal{X}\right),
$$

and go through the proof of theorem 4.2 with $\Gamma_{0}^{\prime}$ instead of $\Gamma_{0}$.

Remark 4.21. Can one prove the commutativity of lemma 4.18 also modulo rational equivalence, i.e. can one prove

$$
\left(L^{2}\right)_{*} \iota^{*} \stackrel{? ?}{=} \iota^{*}\left(L^{2}\right)_{*}: \quad A^{i}(X) \rightarrow A^{i}(X) ?
$$

This would imply that $\iota$ respects the eigenspace decomposition $\Lambda_{\lambda}^{i}$ of [35] (and in particular, that $\iota$ respects the bigraded ring structure $\left.A_{(*)}^{*}(X)\right)$.

The proof of lemma 4.18 given above does not extend to rational equivalence, for the following reason: The quadratic relation (22) still holds modulo rational equivalence [35, Theorem 14.5], and so $L^{\prime}$ satisfies the quadratic relation (26) modulo rational equivalence. However, the unicity result ([35, Proposition 1.3(v)]), that allowed us to conclude from this that $L= \pm L^{\prime}$, is only known modulo homological equivalence.

(This unicity result modulo rational equivalence is conjecturally true, and would follow from the Bloch-Beilinson conjectures [35, Proposition 3.4].) 


\section{COMPLEMENTS}

This section contains some corollaries and extensions of the main result.

Corollary 5.1. Let $S \subset \mathbb{P}^{3}$ be any smooth quartic. Let $X=S^{[2]}$, and let

$$
\iota: X \rightarrow X
$$

be the rational map defined in [1] (cf. remark 2.23). Let $X^{\prime}$ be a hyperkähler fourfold birational to $X$, and let

$$
\iota^{\prime}: X^{\prime} \rightarrow X^{\prime}
$$

be the rational map induced by $\iota$. Then

$$
\begin{aligned}
\left(\iota^{\prime}\right)^{*}=-\mathrm{id}: & A_{(2)}^{i}\left(X^{\prime}\right) \rightarrow A_{(2)}^{i}\left(X^{\prime}\right) \text { for } i=2,4 ; \\
\left(\iota^{\prime}\right)^{*}=\mathrm{id}: & A_{(j)}^{4}\left(X^{\prime}\right) \rightarrow A_{(j)}^{4}\left(X^{\prime}\right) \text { for } j=0,4 .
\end{aligned}
$$

Proof. First, we note that $X^{\prime}$ has an MCK decomposition ([35] or lemma 2.8 above), so the notation $A_{(*)}^{*}\left(X^{\prime}\right)$ makes sense. Since $X$ and $X^{\prime}$ have isomorphic Chow rings [33], it suffices to prove the statement for $X$. Let

$$
\mathcal{S} \rightarrow B_{\rho}, \quad \mathcal{X} \rightarrow B_{\rho}
$$

denote the families of all smooth quartics $S_{b} \subset \mathbb{P}^{3}$, resp. of all Hilbert schemes $X_{b}=\left(S_{b}\right)^{[2]}$. Note that there is an inclusion

$$
B_{\rho} \supset B
$$

where $B$ is as before (parametrizing smooth quartics of Picard number 1 ), and the complement $B_{\rho} \backslash B$ is the union of countably many closed proper subsets (i.e., a very general point of $B_{\rho}$ is in $B)$. Let $\bar{\Gamma}_{\iota} \in A^{4}\left(\mathcal{X} \times_{B_{\rho}} \mathcal{X}\right)$ denote the closure of the graph of the rational map

$$
\iota: \mathcal{X} \rightarrow \mathcal{X}
$$

One can define relative correspondences $\Gamma_{0}, \ldots, \Gamma_{5}$ for this larger family just as in the proof of theorem 4.2. Since the restriction of $\Gamma_{5}$ to the fibre over a very general point of $B_{\rho}$ is rationally trivial, it follows (using [45, Lemma 3.2]) the same is true over every point of $B_{\rho}$, i.e.

$$
\left.\Gamma_{5}\right|_{\left(S_{b}\right)^{4}}=0 \text { in } A^{4}\left(\left(S_{b}\right)^{4}\right) \quad \forall b \in B_{\rho} .
$$

Just as in the proof of theorem 4.2 , one deduces from this that

$$
\left(\iota_{b}\right)^{*}=-\mathrm{id}: \quad A_{(2)}^{2}\left(X_{b}\right) \rightarrow A_{(2)}^{2}\left(X_{b}\right) \quad \forall b \in B_{\rho} .
$$

To prove the result for $A^{4}$, one extends (again using [45, Lemma 3.2]) proposition 4.16 to all of $B_{\rho}$, i.e.

$$
\left(\iota_{b}\right)^{*}\left(l_{b}\right)=l_{b} \text { in } A_{(0)}^{2}\left(X_{b}\right) \quad \forall b \in B_{\rho} .
$$

Then, just as in the proof of theorem 4.15, using the Shen-Vial isomorphism (theorem 2.17), one finds that any $a \in A_{(2)}^{4}\left(X_{b}\right)$ can be written as $a=l_{b} \cdot d$ with $d \in A_{(2)}^{2}\left(X_{b}\right)$, and thus

$$
\left(\iota_{b}\right)^{*}(a)=\left(\iota_{b}\right)^{*}\left(l_{b} \cdot d\right) \stackrel{! !}{=}\left(\iota_{b}\right)^{*}\left(l_{b}\right) \cdot\left(\iota_{b}\right)^{*}(d)=-l_{b} \cdot d \quad \text { in } A_{(2)}^{4}\left(X_{b}\right) \quad \forall b \in B_{\rho} .
$$

(NB: On the boundary $b \in B_{\rho} \backslash B, \iota_{b}$ is not a morphism but only a rational map. Yet, the equality labelled "!!" is still valid since $d \in A_{A J}^{2}\left(X_{b}\right)$; this is thanks to [35, Proposition B.6].) 
Similarly, any $a \in A_{(4)}^{4}\left(X_{b}\right)$ can be written as $a=d_{1} \cdot d_{2}$ with $d_{i} \in A_{(2)}^{2}\left(X_{b}\right)$ (theorem 2.17(i)). Again using [35, Proposition B.6], we find

$$
\left(\iota_{b}\right)^{*}(a)=\left(\iota_{b}\right)^{*}\left(d_{1} \cdot d_{2}\right)=\left(\iota_{b}\right)^{*}\left(d_{1}\right) \cdot\left(\iota_{b}\right)^{*}\left(d_{2}\right)=d_{1} \cdot d_{2} \text { in } A_{(4)}^{4}\left(X_{b}\right) \quad \forall b \in B_{\rho} .
$$

The case $A_{(0)}^{4}$ is easy: $A_{(0)}^{4}\left(X_{b}\right)$ is generated by $\left(l_{b}\right)^{2}$. Letting $\mathcal{L} \in A^{2}(\mathcal{X})$ be the relative cycle restricting to the distinguished class $l_{b} \in A^{2}\left(X_{b}\right)$ on each fibre (as in the proof of theorem 4.15), we know from theorem 4.15 that

$$
\mathcal{L}^{2}-\iota^{*}\left(\mathcal{L}^{2}\right) \in A^{4}(\mathcal{X})
$$

is rationally trivial on a very general fibre $b \in B_{\rho}$. Invoking [45, Lemma 3.2], this implies $\mathcal{L}^{2}-\iota^{*}\left(\mathcal{L}^{2}\right)$ must be rationally trivial on every fibre, i.e.

$$
\left(l_{b}\right)^{2}-\left(\iota_{b}\right)^{*}\left(\left(l_{b}\right)^{2}\right)=0 \text { in } A^{4}\left(X_{b}\right) \quad \forall b \in B_{\rho} .
$$

Corollary 5.2. Let $X=S^{[2]}$, where $S \subset \mathbb{P}^{3}$ is a quartic of Picard number 2 and not containing lines, as in [32, Section 4 Example 2]. Let $g_{\ell} \in \operatorname{Aut}(X)$ be the non-natural automorphism constructed in [32, Lemma 4.6]. Then

$$
\begin{array}{ll}
\left(g_{\ell}\right)^{*}=\mathrm{id}: & A^{4}(X) \rightarrow A^{4}(X), \\
\left(g_{\ell}\right)^{*}=\mathrm{id}: & A_{\text {hom }}^{2}(X) \rightarrow A_{\text {hom }}^{2}(X) .
\end{array}
$$

Proof. The automorphism $g_{\ell}$ is defined as

$$
g_{\ell}:=\left(\iota_{1} \circ \iota_{2}\right)^{\ell} \in \operatorname{Aut}(X),
$$

where $\iota_{1}, \iota_{2}$ are Beauville involutions corresponding to two different embeddings of $S$ in $\mathbb{P}^{3}$. It follows from corollary 5.1 that

$$
\left(\iota_{1} \circ \iota_{2}\right)^{*}=\mathrm{id}: \quad A^{4}(X) \rightarrow A^{4}(X),
$$

hence in particular $g_{\ell}$ acts as the identity on $A^{4}(X)$.

The second assertion follows from the first by a Bloch-Srinivas argument [7].

Let $X$ and $\iota$ be as in theorem 4.1. As noted in the introduction, we are not able to prove the expected equality

$$
\iota^{*}(a) \stackrel{? ?}{=}-a \text { for all } a \in A_{h o m}^{2}(X) .
$$

This is because of the nuisance (already noted in [35]) of having the subgroup $A_{(0)}^{2}(X) \cap A_{\text {hom }}^{2}(X)$ which is conjecturally, but not provably, zero. As shown in the following corollary, at least this nuisance disappears when intersecting with a divisor:

Corollary 5.3. Let $X$ and $\iota$ be as in theorem 4.1. Let $a \in A_{h o m}^{2}(X)$ and $D \in A^{1}(X)$. Then

$$
\iota^{*}(a \cdot D)=-a \cdot \iota^{*}(D) \text { in } A^{3}(X) .
$$

Proof. As shown by Shen-Vial [35, page 7],

$$
\operatorname{Im}\left(A_{(0)}^{2}(X) \cap A_{\text {hom }}^{2}(X) \stackrel{\cdot D}{\rightarrow} A^{3}(X)\right)=0 .
$$

The result now follows from theorem 4.1. 
The quotient of $X$ under the anti-symplectic involution $\iota$ is an EPW sextic ([29], cf. theorem 2.26 above). Since it is a quotient variety, the Chow groups with $\mathbb{Q}$-coefficients form a ring. The following result is about this ring structure:

Corollary 5.4. Let $X$ and $\iota$ be as in theorem 4.1, and let $Y:=X / \iota$. For any $r \in \mathbb{N}$, let

$$
E^{*}\left(Y^{r}\right) \subset A^{*}\left(Y^{r}\right)
$$

be the subring generated by (pullbacks of) $A^{1}(Y)$ and $A^{2}(Y)$. The cycle class map

$$
E^{k}\left(Y^{r}\right) \rightarrow H^{2 k}\left(Y^{r}\right)
$$

is injective for $k \geq 4 r-1$.

Proof. The point is that $X$, and hence also $X^{r}$, has an MCK decomposition [35]. Let $p: X \rightarrow Y$ denote the quotient morphism.

Lemma 5.5. We have

$$
p^{*} A^{2}(Y) \subset A_{(0)}^{2}(X)
$$

Proof. Clearly,

Given $b \in A^{2}(Y)$, let us write

$$
p^{*} A^{2}(Y) \subset A^{2}(X)^{\iota}
$$

$$
p^{*}(b)=c_{0}+c_{2} \quad \in A_{0)}^{2}(X) \oplus A_{(2)}^{2}(X) .
$$

Applying $\iota$, we find

On the other hand,

$$
\iota^{*} p^{*}(b)=c_{0}+c_{2} \quad \in A_{0)}^{2}(X) \oplus A_{(2)}^{2}(X)
$$

$$
\iota^{*} p^{*}(b)=\iota^{*}\left(c_{0}\right)+\iota^{*}\left(c_{2}\right)=\iota^{*}\left(c_{0}\right)-c_{2} \quad \in A_{0)}^{2}(X) \oplus A_{(2)}^{2}(X)
$$

(where we have used corollary 4.19 to obtain that $\iota^{*}\left(c_{0}\right) \in A_{(0)}^{2}(X)$, and theorem 4.2 to obtain that $\left.\iota^{*}\left(c_{2}\right)=-c_{2}\right)$. Comparing these two expressions, we find

$$
\iota^{*}\left(c_{0}\right)=c_{0} \quad \text { in } A_{(0)}^{2}(X), \quad-c_{2}=c_{2} \text { in } A_{(2)}^{2}(X),
$$

proving lemma 5.5.

Lemma 5.5, combined with the obvious fact that $A^{1}(X)=A_{(0)}^{1}(X)$, implies that

$$
\left(p^{r}\right)^{*} E^{*}\left(Y^{r}\right) \subset A_{(0)}^{*}\left(X^{r}\right) .
$$

Since there is a commutative diagram

$$
\begin{array}{rrr}
A_{(0)}^{k}\left(X^{r}\right) & \rightarrow & H^{2 k}\left(X^{r}\right) \\
\uparrow\left(p^{r}\right)^{*} & & \uparrow\left(p^{r}\right)^{*} \\
E^{k}\left(Y^{r}\right) & \rightarrow & H^{2 k}\left(Y^{r}\right)
\end{array}
$$

and the cycle class map

$$
A_{(0)}^{k}\left(X^{r}\right) \rightarrow H^{2 k}\left(X^{r}\right)
$$

is known to be injective for $k \geq 4 r-1$ ([39, Introduction]; this follows for instance from [38, Section 4.3]), this establishes corollary 5.4. 
We single out a particular case of corollary 5.4:

Corollary 5.6. Let $X$ and $\iota$ be as in theorem 4.1, and let $Y:=X / \iota$. The subspaces

$$
\begin{aligned}
& \operatorname{Im}\left(A^{2}(Y) \otimes A^{1}(Y) \rightarrow A^{3}(Y)\right), \\
& \operatorname{Im}\left(A^{2}(Y) \otimes A^{2}(Y) \rightarrow A^{4}(Y)\right)
\end{aligned}
$$

are of dimension 1.

Proof. This follows from corollary 5.4, combined with the fact that

$$
N^{3}(Y):=\left(A^{3}(Y) \rightarrow H^{6}(Y)\right)
$$

is of dimension 1 . To see this, since the pairing

$$
N S(X)^{\iota} \otimes N^{3}(X)^{\iota} \rightarrow N^{4}(X)^{\iota} \cong \mathbb{Q}
$$

is non-degenerate, it suffices to prove

$$
\operatorname{dim} N S(Y)=\operatorname{dim} N S(X)^{\iota}=1 .
$$

But $\operatorname{dim} H^{2}(Y)=1$ (weak Lefschetz for the hypersurface $Y \subset \mathbb{P}^{5}$ ), and so

$$
\operatorname{dim} H^{2}(X)^{\iota}=1 \text {, }
$$

proving (30).

(Alternatively, (30) can also be proven directly: $\iota$ acts on $N S(X)$ as reflection in the span of $D$ (proposition 2.21), and so $N S(X)^{\iota}=\mathbb{Q}[D]$ is of dimension 1.)

Remark 5.7. For any EPW sextic $Y$, conjecturally the cycle class map

$$
E^{k}\left(Y^{r}\right) \rightarrow H^{2 k}\left(Y^{r}\right)
$$

is injective for all $k$ and all $r$ (this is an analogue of the Beauville-Voisin conjecture for powers of K3 surfaces [4], cf. [22]). This is true if $Y$ has an MCK decomposition and, in addition, the conjectural vanishing

$$
A_{(0)}^{2}(Y) \cap A_{h o m}^{2}(Y) \stackrel{? ?}{=} 0
$$

holds.

Also, the pullbacks of the diagonal $\Delta_{Y}$ and the Chern classes $c_{j}(Y)$ should be in the subring $E^{*}\left(Y^{r}\right)$. (I have not been able to prove corollary 5.4 for this larger subring containing pullbacks of $\Delta_{Y}$. The problem is that one needs to prove that $\Gamma_{\iota}$ is in $A_{(0)}^{4}(X \times X)$, i.e. that $\iota$ is "of pure grade 0" in the sense of [36, Definition 1.1].)

Remark 5.8. It is instructive to compare corollary 5.6 with known results concerning the Chow ring of $K 3$ surfaces and of Calabi-Yau varieties. For any $K 3$ surface $S$, it is known that

$$
\operatorname{dim} \operatorname{Im}\left(A^{1}(S) \otimes A^{1}(S) \rightarrow A^{2}(S)\right)=1
$$

[4]. For a generic Calabi-Yau complete intersection $X$ of dimension $n$, it is known that

$$
\operatorname{dim} \operatorname{Im}\left(A^{i}(X) \otimes A^{n-i}(X) \rightarrow A^{n}(X)\right)=1, \quad \forall 0<i<n
$$


[40], [14].

The new part of corollary 5.6, with respect to these results, is the part about

$$
\operatorname{Im}\left(A^{2}(X) \otimes A^{1}(X) \rightarrow A^{3}(X)\right) .
$$

This part is conjecturally true for all EPW sextics [22], but presumably not true for general Calabi-Yau varieties (or even general Calabi-Yau complete intersections). This is related to the question of determining which varieties satisfy Beauville's weak splitting property [3].

We also get the following corollary, providing an alternative description of the Fourier decomposition on $A^{4}(X)$ :

Corollary 5.9. Let $X$ and $\iota$ be as in theorem 4.1, and let $p: X \rightarrow Y:=X / \iota$ be the quotient morphism. Then

$$
\begin{aligned}
& A_{(4)}^{4}(X)=p^{*} A_{\text {hom }}^{4}(Y), \\
& A_{(2)}^{4}(X)=\operatorname{ker}\left(A^{4}(X) \stackrel{p_{*}}{\rightarrow} A^{4}(Y)\right) .
\end{aligned}
$$

Proof. Theorem 4.1 implies that

$$
A_{(4)}^{4}(X)=A^{4}(X)^{\iota} \cap A_{h o m}^{4}(X)
$$

(which proves the first statement of the corollary), and also that

$$
A^{4}(X)=A^{4}(X)^{\iota} \oplus A_{(2)}^{4}(X)
$$

(which proves the second statement of the corollary).

Remark 5.10. Let $X$ and $Y$ be as in corollary 5.9. It seems likely that also

$$
A_{(2)}^{2}(X) \stackrel{? ?}{=} \operatorname{ker}\left(A^{2}(X) \stackrel{p_{*}}{\longrightarrow} A^{2}(Y)\right) .
$$

To prove this, it remains to establish that $\iota$ acts as the identity on $A_{(0)}^{2}(X) \cap A_{\text {hom }}^{2}(X)$ (which is conjecturally 0 ).

\section{OPEN QUESTIONS}

Question 6.1. Let $X$ and $\iota$ be as in theorem 4.1. Can one say anything about the action of $\iota$ on $A^{3}(X)$ ? This seems more difficult than theorem 4.1. Indeed, the action of $\iota$ on $A_{h o m}^{2}$ and on $A^{4}$ is determined by "behaviour up to codimension 1 phenomena". The action of $\iota$ on $A_{(2)}^{3}$, on the other hand, should be determined by the action of $\iota$ on $H^{3,1}(X)$, which is not as neat as the action on $H^{2,0}(X)$ and $H^{4,0}(X)$. I am not even sure what the conjectural statement should be.

Question 6.2. Let $X$ and $\iota$ be as in theorem 4.1. Does the EPW sextic $Y=X / \iota$ have a (selfdual) MCK decomposition? I have not been able to prove this (essentially, this reduces to the problem of showing that $\iota$ is of pure grade 0 , in the sense of [36, Definition 1.1]). 
Question 6.3. As we have seen (theorem 2.20), other cases where a non-symplectic, non-natural involution exists on $S^{[2]}$ is when $S$ is a generic $K 3$ surface of degree $d=20,26,34, \ldots$ (i.e., of genus $g=11,14,18, \ldots)$. It would be interesting to prove Bloch's conjecture for these cases as well.

For the case $d=34$ (i.e., $g=18$ ), Mukai [24] has given a nice description of $S$ in terms of sections of a vector bundle on an orthogonal Grassmannian, so there is at least some hope that the method of spread à la Voisin can be employed in this case as well. Let $\mathcal{S} \rightarrow B$ be the family of all smooth dimension 2 sections of this vector bundle. One major difficulty is in proving a version of theorem 4.5 for the fourfold relative fibre product of this family $\mathcal{S} \rightarrow B$, i.e. one would need to prove

$$
A_{\text {hom }}^{2}\left(\mathcal{S}^{4 / B}\right)=0
$$

Is this feasible?

Question 6.4. Let $S$ be a generic $K 3$ surface of degree $d=10$ (i.e., of genus $g=6$ ). The Hilbert scheme $X=S^{[2]}$ has no non-trivial automorphisms (theorem 2.20), but there is a nonsymplectic rational involution

$$
\iota: X \rightarrow X
$$

constructed by O'Grady [28, Section 4.3]. Can one prove the statement of theorem 4.1 in this set-up ? Work of Mukai [23] realizes these K3 surfaces as complete intersections in a certain Grassmannian. Again, the main difficulty seems to consist in proving that

$$
A_{\text {hom }}^{2}\left(\mathcal{S}^{4 / B}\right)=0
$$

for this family. Is this feasible?

Question 6.5. It would also be interesting to extend theorem 4.1 to higher dimensional Hilbert schemes $S^{[r]}, r>2$. Let $S \subset \mathbb{P}^{r+1}$ be a K3 surface of degree $2 r$. The Hilbert scheme $S^{[r]}$ has an MCK decomposition [39], and so there is a bigraded ring structure $A_{(*)}^{*}\left(S^{[r]}\right)$. As noted by Beauville [1], there is a non-trivial rational involution

$$
\iota: S^{[r]} \rightarrow S^{[r]} \text {. }
$$

Can one prove something about the action of $\iota$ on $A_{(*)}^{*}\left(S^{[r]}\right)$ ? Supposing one wants to follow the approach of the present article, the main difficulty consists in proving that

$$
A_{\text {hom }}^{2}\left(\mathcal{S}^{2 r / B}\right)=0
$$

(or even $\operatorname{Griff}^{2}\left(\mathcal{S}^{2 r / B}\right)=0$ ), where $\mathcal{S} \rightarrow B$ is the family of all smooth $K 3$ surfaces of degree $2 r$ in $\mathbb{P}^{r+1}$.

Acknowledgements. Thanks to all participants of the Strasbourg 2014/2015 "groupe de travail" based on the monograph [45] for a stimulating atmosphere. Many thanks to Yasuyo, Kai and Len for efficiently managing the Schiltigheim Math Research Institute. 


\section{REFERENCES}

[1] A. Beauville, Some remarks on Kähler manifolds with $c_{1}=0$, in: Classification of algebraic and analytic manifolds (Katata, 1982), Birkhäuser Boston, Boston 1983,

[2] A. Beauville, Variétés Kähleriennes dont la première classe de Chern est nulle, J. Differential Geom. 18 no. 4 (1983), 755-782,

[3] A. Beauville, On the splitting of the Bloch-Beilinson filtration, in: Algebraic cycles and motives (J. Nagel and C. Peters, editors), London Math. Soc. Lecture Notes 344, Cambridge University Press 2007,

[4] A. Beauville and C. Voisin, On the Chow ring of a K3 surface, J. Alg. Geom. 13 (2004), 417—426,

[5] S. Bloch, Lectures on algebraic cycles, Duke Univ. Press Durham 1980,

[6] S. Bloch, A. Kas and D. Lieberman, Zero cycles on surfaces with $p_{g}=0$, Comp. Math. 33 no. 2 (1976), 135-145,

[7] S. Bloch and V. Srinivas, Remarks on correspondences and algebraic cycles, American Journal of Mathematics Vol. 105, No 5 (1983), 1235-1253,

[8] S. Boissière, A. Cattaneo, M. Nieper-Wißkirchen and A. Sarti, The automorphism group of the Hilbert scheme of two points on a generic projective $K 3$ surface, arXiv:1410.8387v2, in: K3 Surfaces and Their Moduli, Proceedings of the Schiermonnikoog conference 2014 (C. Faber, G. Farkas, G. van der Geer, editors), Progress in Maths 315, Birkhäuser 2016,

[9] S. Boissière and A. Sarti, A note on automorphisms and birational transformations of holomorphic symplectic manifolds, Proc. Amer. Math. Soc. 140 (2012), 4053-4062,

[10] M. de Cataldo and L. Migliorini, The Chow groups and the motive of the Hilbert scheme of points on a surface, Journal of Algebra 251 no. 2 (2002), 824 - 848,

[11] A. Corti and M. Hanamura, Motivic decomposition and intersection Chow groups, I, Duke Math. J. 103 (2000), 459-522,

[12] C. Deninger and J. Murre, Motivic decomposition of abelian schemes and the Fourier transform. J. reine u. angew. Math. 422 (1991), 201-219,

[13] D. Eisenbud, S. Popescu and C. Walter, Lagrangian subbundles and codimension 3 subcanonical subschemes, Duke Math. J. 107(3) (2001), 427-467,

[14] L. Fu, Decomposition of small diagonals and Chow rings of hypersurfaces and Calabi-Yau complete intersections, Advances in Mathematics (2013), 894-924,

[15] L. Fu, On the action of symplectic automorphisms on the $C H_{0}$-groups of some hyper-Kähler fourfolds, Math. Z. 280 (2015), 307-334,

[16] W. Fulton, Intersection theory, Springer-Verlag Ergebnisse der Mathematik, Berlin Heidelberg New York Tokyo 1984,

[17] D. Huybrechts, Symplectic automorphisms of $K 3$ surfaces of arbitrary order, Math. Res. Letters 19 (2012), 947-951,

[18] U. Jannsen, Motivic sheaves and filtrations on Chow groups, in: Motives (U. Jannsen et alii, eds.), Proceedings of Symposia in Pure Mathematics Vol. 55 (1994), Part 1,

[19] U. Jannsen, Equivalence relations on algebraic cycles, in: The arithmetic and geometry of algebraic cycles (B. Gordon et alii, eds.), Banff Conference 1998, Kluwer,

[20] U. Jannsen, On finite-dimensional motives and Murre's conjecture, in: Algebraic cycles and motives (J. Nagel and C. Peters, editors), Cambridge University Press, Cambridge 2007,

[21] B. Kahn, J. Murre and C. Pedrini, On the transcendental part of the motive of a surface, in: Algebraic cycles and motives (J. Nagel and C. Peters, editors), Cambridge University Press, Cambridge 2007,

[22] R. Laterveer, Algebraic cycles on a very special EPW sextic, submitted,

[23] S. Mukai, Curves, K3 surfaces and Fano 3-folds of genus $\leq 10$, in: Algebraic Geometry and Commutative Algebra I, Kinokuniya Tokyo 1988,

[24] S. Mukai, Polarized K3 surfaces of genus 18 and 20, in: Complex projective geometry (Trieste, 1989/Bergen, 1989), London Math. Soc. Lecture Note Ser. 179, Cambridge Univ. Press, Cambridge 1992, 
[25] J. Murre, On a conjectural filtration on the Chow groups of an algebraic variety, parts I and II, Indag. Math. 4 (1993), 177-201,

[26] J. Murre, J. Nagel and C. Peters, Lectures on the theory of pure motives, Amer. Math. Soc. University Lecture Series 61, Providence 2013,

[27] J. Nagel and M. Saito, Relative Chow-Künneth decompositions for conic bundles and Prym varieties, Int. Math. Res. Not. 2009, no. 16 (2009), 2978-3001,

[28] K. O'Grady, Involutions and linear systems on holomorphic symplectic manifolds, Geom. Funct. Anal. 15 no 6 (2005), 1223-1274,

[29] K. O'Grady, Irreducible symplectic 4-folds and Eisenbud-Popescu-Walter sextics, Duke Math. J. 134(1) (2006), 99-137,

[30] K. O’Grady, EPW-sextics: taxonomy, Manuscripta Math. 1381 (2012), 221—272,

[31] K. O'Grady, Double covers of EPW-sextics, Michigan Math. J. 62 (2013), 143-184,

[32] K. Oguiso, A remark on dynamical degrees of automorphisms of hyperkähler manifolds, Manuscripta Math. 130 (2009), 101-111,

[33] U. Rieß, On the Chow ring of birational irreducible symplectic varieties, Manuscripta Math. 145 (2014), $473-501$,

[34] T. Scholl, Classical motives, in: Motives (U. Jannsen et alii, eds.), Proceedings of Symposia in Pure Mathematics Vol. 55 (1994), Part 1,

[35] M. Shen and C. Vial, The Fourier transform for certain hyperKähler fourfolds, Memoirs of the AMS 240 (2016), no.1139,

[36] M. Shen and C. Vial, The motive of the Hilbert cube $X^{[3]}$, arXiv:1503.00876,

[37] C. Vial, Remarks on motives of abelian type, to appear in Tohoku Math. J.,

[38] C. Vial, Niveau and coniveau filtrations on cohomology groups and Chow groups, Proceedings of the LMS 106(2) (2013), 410—444,

[39] C. Vial, On the motive of some hyperkähler varieties, to appear in J. für Reine u. Angew. Math.,

[40] C. Voisin, Chow rings and decomposition theorems for $K 3$ surfaces and Calabi-Yau hypersurfaces, Geom. Topol. 16 (2012), 433-473,

[41] C. Voisin, Symplectic involutions of $K 3$ surfaces act trivially on $C H_{0}$, Documenta Math. 17 (2012), $851-860$,

[42] C. Voisin, The generalized Hodge and Bloch conjectures are equivalent for general complete intersections, Ann. Sci. Ecole Norm. Sup. 46, fascicule 3 (2013), 449-475,

[43] C. Voisin, The generalized Hodge and Bloch conjectures are equivalent for general complete intersections, II, J. Math. Sci. Univ. Tokyo 22 (2015), 491—517,

[44] C. Voisin, Bloch's conjecture for Catanese and Barlow surfaces, J. Differential Geometry 97 (2014), $149-175$,

[45] C. Voisin, Chow Rings, Decomposition of the Diagonal, and the Topology of Families, Princeton University Press, Princeton and Oxford, 2014,

[46] C. Voisin, Remarks and questions on coisotropic subvarieties and 0-cycles of hyper-Kähler varieties, in: K3 Surfaces and Their Moduli, Proceedings of the Schiermonnikoog conference 2014 (C. Faber, G. Farkas, G. van der Geer, editors), Progress in Maths 315, Birkhäuser 2016.

Institut de Recherche Mathématique Avancée, CNRS - Université de Strasbourg, 7 Rue RENÉ Descartes, 67084 Strasbourg CEDEX, FRANCE.

E-mail address: robert. laterveer@math.unistra.fr 